\title{
Paläohydrologische Untersuchungen zur Umlenkung der Radolfzeller Aach bei Rielasingen-Arlen
}

\author{
Von Hubert Blum, Wolfgang Bludau, \\ WOLFGANG OSTENDORP \& EDITH SCHMIDT ${ }^{1}$
}

Mit 9 Abbildungen und 4 Tabellen

\begin{abstract}
Zusammenfassung
Durch eine Kombination von geomorphologischen, feldgeologischen und sedimentologischen Methoden konnten die paläohydrologischen Ereignisse, die mit der Laufrichtungsänderung der Radolfzeller Aach im Bereich Rielasingen in Zusammenhang stehen, weitgehend aufgeklärt werden:

1. Die morphologischen Befunde bestätigen, daß sich im Bereich Rielasingen-Arlen der Auslauf des Eisrandstausees befand, der sich in der Zeit zwischen den Gletscherrückzugsständen 7 und 8 im westlichen Unterseegebiet gebildet hatte.

2. Die Untere Radolfzeller Aach nahm ursprünglich einen anderen Verlauf als heute: Sie floß von Rielasingen aus in südwestlicher Richtung, erreichte westlich der Ortschaft Ramsen das untere Bibertal und folgte diesem bis zur Mündung in den Hochrhein, etwa $2 \mathrm{~km}$ westlich von Hemishofen. Damit ist der heute ab Rielasingen-Arlen erscheinende Ostverlauf sekundärer Natur und wurde durch eine Ablenkung des alten Gerinnes verursacht. Indizien für einen mehrfachen Wechsel zwischen dem Bodensee- und Hochrhein-Mündungsgebiet liegen nicht vor.

3. Die Laufrichtungsänderung fand im Zeitabschnitt Allerød/Jüngere Dryas statt (ca. $11000 \mathrm{BP} ;{ }^{14} \mathrm{C}$-Daten und pollenanalytische Datierungen).

4. Östlich des Aachknies können 3 Flußrinnen ausgeschieden werden: Die als „Ostrinne I" bezeichnete Rinne dürfte bis etwa $1000 \mathrm{BC}$ aktiv gewesen sein. Die Ostrinne II liegt etwas nördlich der ersten; eine Datierung der Inaktivierung war hier nicht möglich. Die dritte, nördlichste Rinne, entspricht der heutigen Situation.

${ }^{1}$ Anschriften der Verfasser: Dipl.-Geogr. H. BLum, Landesdenkmalamt Bad.Württ., Fischersteig 9, D-78343 Gaienhofen. Dipl.-Biol. Dr. W. Bludau, Geologisches Landesamt Bad.-Württ., Kaiserstuhlstr. 28, D-79106 Freiburg. Dipl.-Biol. Dr. W. OstendorP, Fakultät für Biologie der Universität, D-78434 Konstanz. Dipl.-Biol. E. Schmid, Zoologisches Institut der Universität, Albertstr. 23a, D-79104 Freiburg.
\end{abstract}


5. Das Fehlen jeglicher paläohydrologischer Spuren aus dem frühen und mittleren Holozän wird damit erklärt, daß infolge der damals dichten Waldbedeckung eine hydrologisch stabile Phase angenommen werden kann, innerhalb derer die Hochwasserscheitel schwächer ausfielen. Damit kann für diesen Zeitabschnitt die Neigung zu Gerinnebettveränderungen eher gering eingeschätzt werden.

\section{Einleitung}

Die Radolfzeller Aach, mit einer mittleren Wasserflihrung von etwa $10 \mathrm{~m}^{3} \mathrm{~s}^{1}$ (Mündung), nach dem Seerhein der bedeutendste oberirdische Zufluß des Bodensee-Untersees, entspringt unmittelbar nördlich der Ortschaft Aach (Landkreis Konstanz) als Karstquelle, die erst mit dem Rückgang des würmzeitlichen Rheingletschers freigelegt wurde (Abb. 1). Auf seinem Weg nach Süden folgte der Fluß zunächst der Schmelzwasservorflut, so etwa über das Singener Schotterfeld (Rückzugsstände 6 und 7). das mit dem Zurückweichen der Gletscherfront zum Stand 8 (Böhringer Terrasse) vollständig trockenfiel (Abb. 2). ERB (1949) geht davon aus. daß die Radolfzeller Aach zunächst über Rielasingen und Ramsen hinaus dem Weg der Schmelzwasserabflüsse der Stände 7 und 8 bis zu ihrer Mündung in den Hochrhein folgte (Abb. 1).

Heute hingegen liegt das ca. $300 \mathrm{~m}$ breite glazialzeitliche Tal zwischen Rielasingen-Arlen und Ramsen trocken; nur der Abschnitt südlich von Ramsen wird heute noch von dem Flüßchen Biber als Abflußrinne benutzt. Die Radolfzeller Aach selbst hat im Gebiet von Rielasingen/Arlen abrupt ihre Laufrichtung geändert, indem sie sich nach Osten. dem Lintersee-Becken zuwendet und zwischen Moos und Radolfzell in den Zeller See. einem Teilbecken des Untersees, mündet. Das sog. „Aachknie“ bei Rielasingen hat bereits früh das Interesse der Quartärgeologen hervorgerufen. SCHMIDLE (1906:89 und 1914:70) kam zu dem Schluß, daß der Fluß sekundär in die Depression des westlichen Zeller-See-Beckens eingedrungen sein muB: als Ursache für die angenommene Laufrichtungsänderung diskutiert er tektonische Vorgänge. ERB (1949) zieht zur Erklärung der Ablenkung vorwiegend morphologische Aspekte heran, denn moderne Datierungstechniken standen ihm seinerzeit noch nicht zur Verfügung. Eine grobe relative Zeitangabe des vermuteten Ereignisses wird aus dem Altersvergleich mit einer von der Aach teilweise erodierten archäologischen Fundstelle. die mesolithische Kulturreste enthalten haben soll, hergeleitet. Schlichtherle (1985:27) bringt die Laufänderung der Aach in Zusammenhang mit Wasserspiegelschwankungen des Bodensees, indem er auf die Möglichkeit hindeutet, daß im Bereich der vermuteten ehemaligen Mündung westlich Hemishofen als Folge des Sedimenteintrages durch die Aach eine Auflandung der Hochrheinsohle stattgefunden haben könnte, was wiederum 


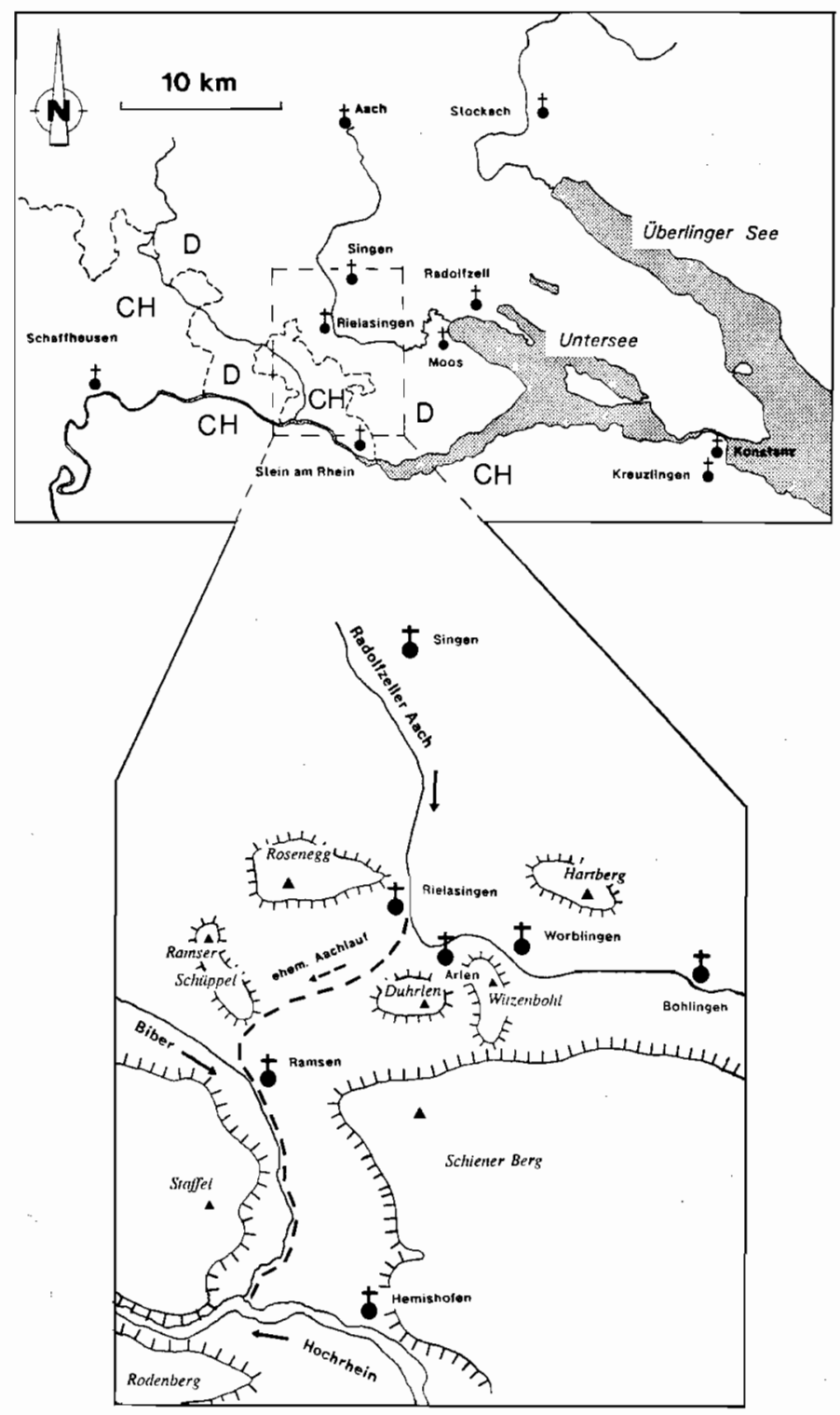

Abb. 1: Übersicht über das westliche Bodenseegebiet sowie des Untersuchungsgebietes zwischen Singen, Ramsen und Bohlingen. 
durch Rückstau zwangsläufig zu einem erhöhten Seespiegel im Untersee hätte führen müssen.

Die vorliegende Arbeit versucht nun mit modernen paläohydrologischen Methoden zu klären, welche Wege die Aach südlich von Singen tatsächlich jemals genommen hat, und wann, bzw. unter welchen Begleitumständen eine Laufrichtungsänderung eingetreten ist. Wichtigste Hilfsmittel sind das Abbohren von talquerenden Transekten, die Öffnung, Feinstratifizierung und Beprobung von Baggerschürfen, Korngrößenanalyse der ergrabenen Sedimente und als Datierungsmittel die Kombination von Pollenanalyse und Radiokarbonaltersbestimmung.

Das im Landkreis Konstanz gelegene Untersuchungsgebiet umfaßt den südöstlichen Abschnitt des Blattes 8218 (Gottmadingen) sowie den südwestlichen Teil des Blattes 8219 (Singen) der TK 25 (vgl. Abb. 1). Es erstreckt sich, jeweils ausgehend vom Aachknie, entlang der heutigen Flußniederung bis zum Ortsrand von Rielasingen im Norden und bis zur Ortsmitte von Worblingen im Osten. Im SW folgt es dem in Richtung Ramsen ziehenden Trockental bis zur Bundesgrenze.

Eine ausführliche Schilderung der Geologie und der pleistozänen landschaftsgeschichtlichen Entwicklung findet sich bei SchreINer (1974 u. 1989).

\section{Methoden}

Geländeaufnahme, Bohrprogramm und Bohrtechnik: Im Rahmen einer breit angelegten, methodisch den Zielen dieser Untersuchung angepaßten geomorphologischen Kartierung wurden zahlreiche Strukturen beobachtet, die auf fossile Rinnenelemente hindeuten (BlcM 1993). Auf der Grundlage dieser Ergebnisse wurden 6 talquerende Bohrtransekte festgelegt, lage- und höhenmäßig eingemessen und im $10 \mathrm{~m}$-Abstand mit leichtem Handgerät (Bohrstock 1,7 m lang, bzw. Pürckhauer-Bohrer mit max. $4 \mathrm{~m}$ Verlängerungsgestänge) abgebohrt. An besonders interessierenden Positionen wurden ergänzend von einer Fachfirma 4 Rammkernbohrungen niedergebracht. Darüberhinaus wurden mit Hilfe eines Mittelbaggers insgesamt 21 Gruben vom Ausmaß 2 auf $1,5 \mathrm{~m}$ und ca. $2 \mathrm{~m}$ Tiefe geöffnet. Ausgewählt wurden Positionen, die entweder bereits durch ihre Morphologie aufgefallen waren oder denen aufgrund der Bohrbefunde größere Bedeutung zugemessen worden war. Ziel der Baggerschürfe war die feinstratigraphische Aufnahme der Schicht- und Lagerungsverhältnisse und die Gewinnung von Materialproben für die sedimentologischen Lntersuchungen, die pollenanalytischen Auswertungen und die ${ }^{14} \mathrm{C}$-Datierungen. 


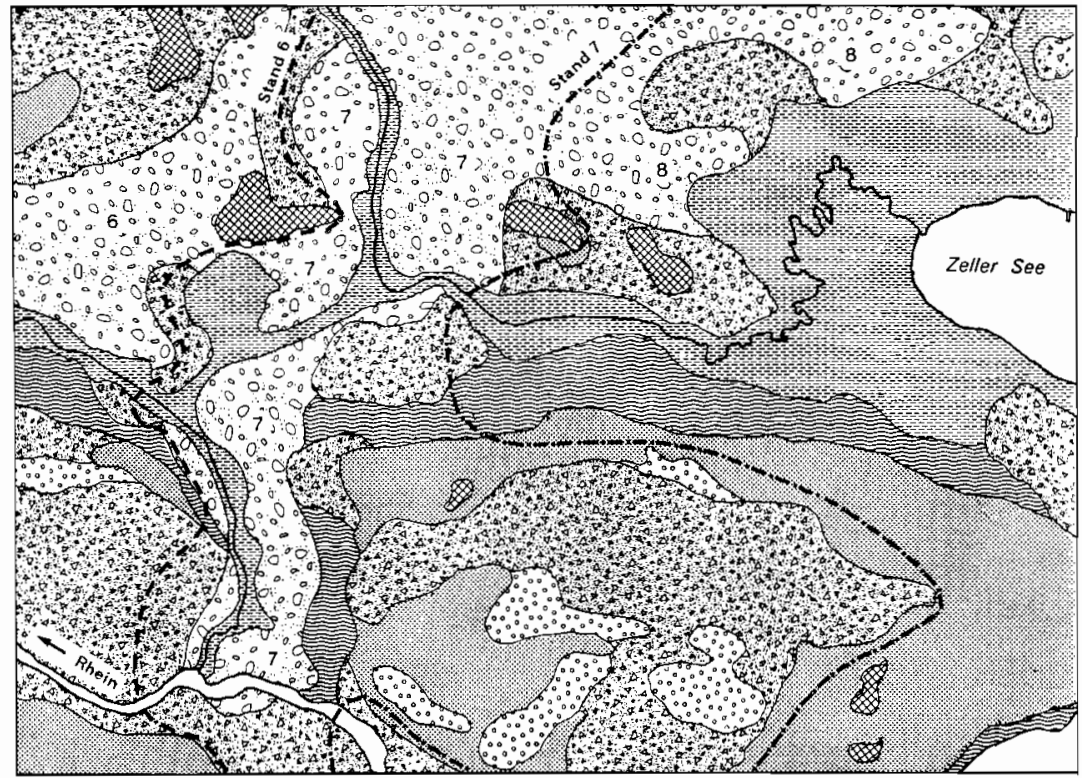

Geologische Übersichtskarte

$5 \mathrm{~km}$

Untere Radolfzeller Aach / Schiener Berg

\section{Quartär}

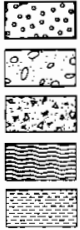

Tertiär

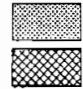

Obere SüB wassermolasse

Deckentuffe

Würmschotter

Grundmoräne

Schwemmlehm

Jallehme/Torfe

Abb. 2: Geologische Übersicht des Untersuchungsgebietes und seiner näheren Umgebung. Gekennzeichnet sind die Schotterfelder der Rückzugsstände 6 bis 8 des westl. Rheinvorlandgletschers. (verändert n. ScHREINER 1989). 
Die Bezeichnung der einzelnen Transekte, Gruben, Proben und lithologischen Einheiten folgt der nachstehenden Systematik (zur topogr. Lage der Transekte vgl. Abb. 3 und 4):

- Die Bohrtransekte orientieren sich am heutigen Aachknie als Nullpunkt und sind in der jeweiligen Himmelsrichtung aufsteigend durchnumeriert (Bspl.: T3 SW = dritter Transekt südwestlich des Aachknies)

- Ein „G“ steht für den auf den entsprechenden Transekt bezogenen Grubenkomplex; der nachgestellte Großbuchstabe markiert die einzelne Grube (Bspl:: G2SW/B = Grubenkomplex am T2 SW, Grube B).

- Materialproben erhalten die Einzelgrubenbezeichnung plus die lithologische Einheit (LE). (Bspl.: G1E/A-4.1.2= Grubenkomplex am T1 E, Grube A, lithologische Haupteinheit 4, lithologische Untereinheit 1.2).

- „K" steht für „Kurzkern“; die nachgestellten römischen Zahlen sind vom Hangenden zum Liegenden aufsteigend geordnet. (Bspl.: G2SW/ A-KII = Entnahmegrube G2SW/A, zweiter Kurzkern von oben).

- Die Rammkernbohrungen werden mit einem vorangestellten „B“ gekennzeichnet (Bspl. B3SW/A = Bohrung A am dritten SW-Transekt).

Probennahme: Das Probenmaterial (genau stratifizierte Mischproben von etwa 5 bis $15 \mathrm{~kg}$ Frischsubstanz [FS]) wurde aus den gesäuberten Profilwänden der Baggerschürfen entnommen. Feinkörnige Lockersedimente wurden darüberhinaus mit Kunststoffrohren $(50 \mathrm{~cm} \times 10 \mathrm{~cm} \varnothing)$ durchteuft, um feinstratigraphisch ungestörtes Material für die Pollenanalyse zu gewinnen. Insgesamt wurden 10 derartige „Kurzkerne“ geborgen. Die Kerne wurden im Labor der Länge nach aufgesägt und geteilt, lithologisch stratifiziert, fotographiert und beprobt (i.a. Pollenproben mit einem Standardprobenabstand von $10 \mathrm{~cm}$ ).

Korngrößen analysen: Die o.g. Mischproben wurden in bergfrischem Zustand ohne chemischen Aufschluß und ohne Abtrennung der organischen Substanz granulometrisch analysiert. Die Kies-Fraktionen $(\varnothing>2,0$ $\mathrm{mm})$ wurden von Hand, die Sand-Fraktionen $(\varnothing=0,063 \ldots 2,0 \mathrm{~mm}) \mathrm{mit}$ Hilfe einer 3D-Vibrationssiebmaschine durch die entsprechenden Siebe geführt. Der Durchlauf des $0,063 \mathrm{~mm}$-Siebes wurde aufgefangen und diente als Einsatz für die laseroptische Korngrößenbestimmung im Particle Analyzer (Typ CIS-1 der Fa. Galai, Tel Aviv, Israel) am Institut für Seenforschung, Langenargen (zur Granulometrie vgl. OstendoRP 1995). Da Korngrößenverteilungen aufgrund der großen Spannweite der Teilchendurchmesser in der Regel semilogarithmisch dargestellt werden, dient der $\zeta$-Wert $\left(\zeta:=1 \mathrm{~g}\left(\mathrm{~d} / \mathrm{d}_{\mathrm{O}}\right)\right.$ mit $\mathrm{d}_{\mathrm{O}}=2 \mathrm{~mm},(\mathrm{~d}:$ Korndurchmesser in $[\mathrm{mm}])$ als Grundlage für alle statistischen Berechnungen. Dieser Einteilung entspricht auch die Festlegung der Siebmaschenweiten nach DIN 4220. 
Geoche mie: Eine Teilstichprobe der Mischprobe wurde getrocknet, in einer Kugelmühle homogenisiert und auf $2 \mathrm{~mm}$ abgesiebt. Vom Siebdurchgang wurden ca. $300 \mathrm{mg}$ eingewogen und $8 \mathrm{Std}$. bei $560^{\circ} \mathrm{C}$ im Muffelofen geglüht. Der Glühverlust dient als Maß für den Gehalt an organischer Substanz. Alkali- und Erdalkali-Ionen wurden aus dem HCl-sauren Aufschluß flammen-absorptionsspektrometrisch bestimmt (vgl. OSTENDORP 1995). Der Gesamtkarbonat-Gehalt wurde rechnerisch anhand der Erdalkali-Gehalte ermittelt.

Pollenpräparation und Darstellung im Pollendiagramm: Die Aufbereitung der Proben für die Pollenanalyse erfolgte im Pollenlabor der Universität Hohenheim gemäß eines modifizierten Verfahrens nach Frenzel (1964). Proben aus rein organischer Substanz wurden nacheinander einer $\mathrm{NaOH}$ - und ggf. einer HF-Behandlung unterzogen, anschließend in Eisessig dehydriert und durch Acetolyse nach ERDTMAn et al. (1961) gereinigt. Mineralische Proben wurden durch HCl-Behandlung entkalkt; nach $\mathrm{NaOH}-\mathrm{Behandlung}$ wurden organische und mineralische Reste durch Natrium-Polywolframat-Dichtetrennung $\left(\rho=2,2 \mathrm{~g} \cdot \mathrm{cm}^{-3}\right)$ voneinander getrennt. Der Überstand wurde gewaschen und danach wie oben beschrieben behandelt. Die acetolysierten Proben wurden in Glycerin überführt und in luftdicht verschließbaren Kunststoffröhrchen aufbewahrt. Die Darstellung der Ergebnisse erfolgt in Form vereinfachter Pollendiagramme, welche nur die wichtigsten zur Datierung notwendigen Pollentypen enthalten. Die Berechnungsgrundlage ist die Gesamtpollensumme (GPS $=100 \%$ ). Die Benennung und die chronologische Abgrenzung der Pollenzonen (PZ) erfolgt nach Firbas (1949), modifiziert nach LANG (1990).

${ }^{14} \mathrm{C}$-Datierung: Als Materialien für die Radiokarbondatierung wurden stratifiziert entnommene Stamm- und Astholzreste ausgewählt; Wurzelholz und nicht eindeutig bestimmbare Reste wurden verworfen. Die Holzartbestimmung wurde freundlicherweise von A. BillamBoz beim Landesdenkmalamt Baden-Württemberg in Hemmenhofen durchgeführt. Bei größeren Ästen bzw. Stämmen wurde das Material rindentangential abgeschält um die Präzision der ${ }^{14} \mathrm{C}$-Bestimmung zu erhöhen. Die Altersbestimmung (Zählrohr-Methode) wurde von B. KROMER am Institut für Umweltphysik, Univ. Heidelberg, durchgeführt. Die unkalibrierten ${ }^{14} \mathrm{C}$-Alter werden mit dem Suffix „BP“ (years before present, d.h. Jahre vor 1950) angegeben, kalibrierte ${ }^{14} \mathrm{C}-J a h r e$ (entsprechen Sonnenjahren) tragen die Endungen „BC“ (before Christ) bzw. „AD“ (anno Domini) (vgl. Strahm 1985:252). Die Kalibrierung der ${ }^{14} \mathrm{C}$-Rohdaten wurde mit Hilfe des Programmes CALIB 3.0 vorgenommen (vgl. BeCKER 1993 und Reimer \& STUiver 1993). 


\section{Ergebnisse}

\subsection{Morphologie und Stratigraphie der Talquerschnitte}

Die in den Abb. 5 a-c dargestellen stratigraphischen Talquerprofile beruhen vorwiegend auf den Auswertungen der Handsondagen, teilweise aber auch auf den Ergebnissen der Baggerschürfe sowie der Rammkernbohrungen.

Transekt 1 Nord: Die Abb. 5 a zeigt einen knapp $200 \mathrm{~m}$ breiten Gesamttalquerschnitt, in dem neben der heutigen Aachrinne noch weitere 3 verlandete Rinnen auszumachen sind. Eingebettet sind diese in eine etwa $1,5 \mathrm{~m}$ mächtige Schicht von oberflächlich stark anmoorigem, heterogenem Material, welches, abgesehen von eingelagerten Grobklastika, lehmartigen Charakter aufweist. Aufgrund der sehr flach verlaufenden Schichtoberfläche ist anzunehmen, daß sich vor der anthropogenen Laufkorrektion im 19. Jh. die Flußaue über die gesamte Talsohle erstreckte. Die eigentliche Basis des Tales wird von glazifluvialen Grobkiesen gebildet. Auffallend ist die steile Westflanke: Da direkt unterhalb des Hangfußes eine verlandete Rinne liegt, dürfte diese Querschnittsasymmetrie auf ehemalige Prallhangerosion zurückzuführen sein. Die Querschnittsflächen der einzelnen Rinnen wären, sofern man sie bis zur heutigen Geländeoberfläche extrapoliert und damit die Überdeckung als spätere Bildung ansieht, mit derjenigen der heutigen Aachrinne zu vergleichen. Außerdem weisen die Sohlniveaus aller Rinnen kaum Höhenunterschiede bezüglich einer gedachten Ausgleichshorizontalen auf. Das deutet darauf hin, daß in der Abfolge nur geringfügige Auflandungs- bzw. Tiefenerosionsprozesse erfolgt sind, so daß die Laufverlagerungen durch Mäandrierungen entstanden sein müssen. Weiterhin ist aufgrund dieses Befundes zu vermuten, daß die Altersunterschiede zwischen den Einzelrinnen relativ gering sind. Diese, rein auf die Morphologie gestützte Annahme ist derzeit allerdings noch nicht durch Radiokarbon- oder Pollendatierungen belegt.

Transekt 2 Südwest: Dieses heute trockenliegende Tal ist im Vergleich zu T1 N sowohl deutlich breiter, als auch tiefer eingeschnitten. Die Flanken verlaufen asymmetrisch, wobei die auf der NW-Seite gelegene deutlich versteilt ist. Dieser Asymmetrie entspricht auch der interne Schichtaufbau. An die NW-Seite angrenzend ist eine mit Feinsedimenten verfüllte Struktur (zwischen $X+70$ und $X+100 \mathrm{~m}$ ) um bis zu 4 m eingetieft. Sie wird im folgenden als Hauptrinne bezeichnet. Am Fuße der südöstlichen flacheren Flanke liegt eine etwa 1,5 $\mathrm{m}$ tiefe, mit homogenem Glimmersand gefüllte Nebenrinne (bei X+320 m). Das Basismaterial der gesamten Talsohle ist zweigeteilt: Im nordwestlichen und im zentralen Bereich stehen glaziflu- 


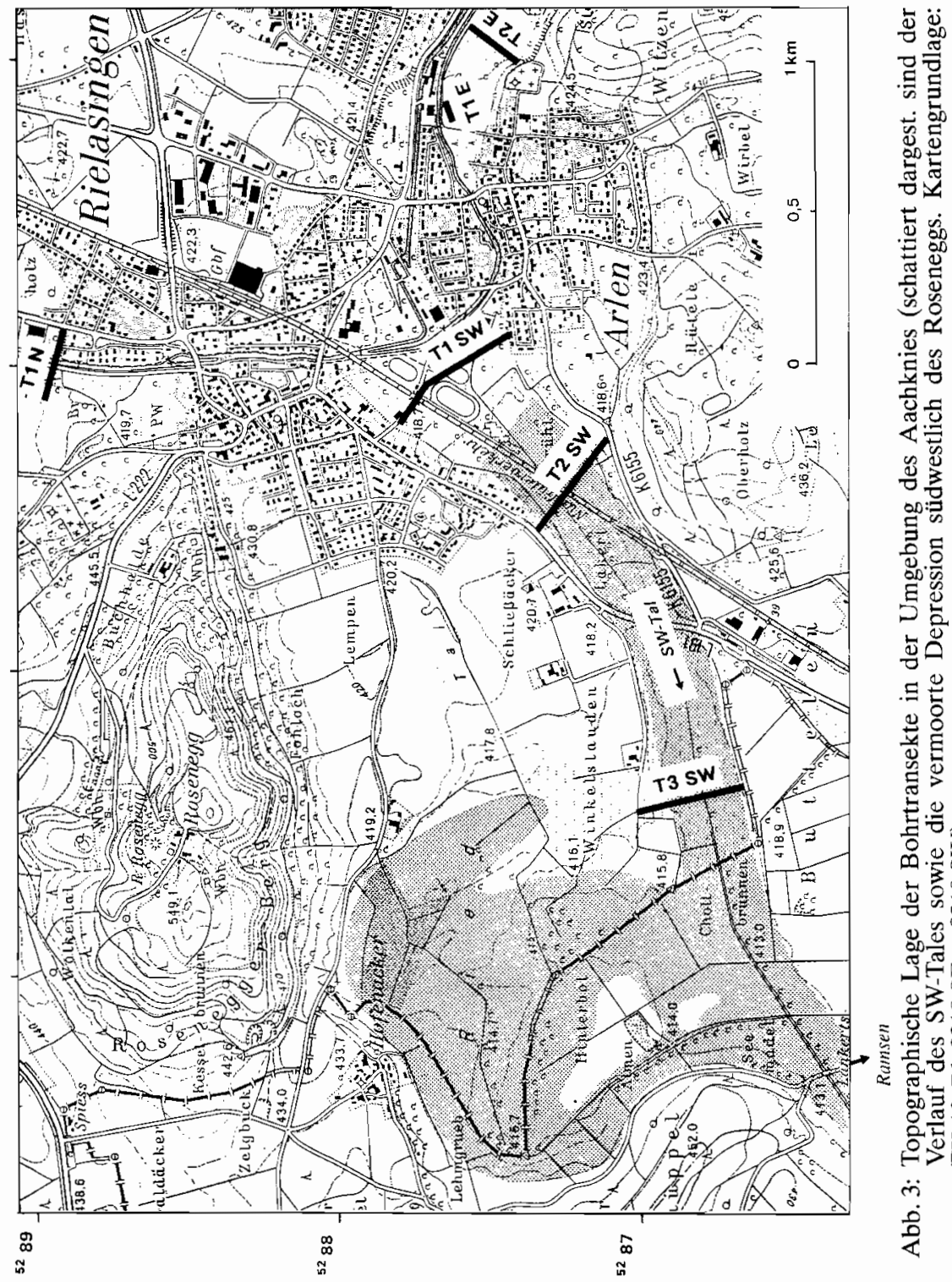


viale Grobkiese an. Im südöstlichen Abschnitt wird die Grundlage von sehr kompakt gelagerter Grundmoräne gebildet. Die feinsedimentäre Auflage beträgt, abgesehen von den Rinnenbereichen, etwa $0,5 \mathrm{~m}$. In Rinnennähe ändert sich die Fazies dieser Auflage insofern, als daß schluffige Abschnitte mit hohem organischem Gehalt ausgeschieden werden können. Es handelt sich dabei um Auelehme. Die oberste Schichtlage besteht aus Viedermoortorf. Zwischen $\mathrm{X}+100 \mathrm{~m}$ und $\mathrm{X}+130 \mathrm{~m}$ treten gestörte Schichtverhältnisse auf, die auf den Bau der Abwassersammelleitung Singen-Ramsen zurückgehen. Die Sandlage bei X+410 m dürfte nicht mehr im Zusammenhang mit dem fluvialen Geschehen im Bereich der Talsohle stehen; sowohl von der Höhenlage als auch von der Materialzusammensetzung her (Glimmersand der jungtertiären Oberen Süßwassermolasse) ist sie dem unter der nach SE ansteigenden Grundmoräne verborgenen Molassekern zuzuordnen. Es dürfte sich mit hoher Wahrscheinlichkeit um älteres Abschwemmungsmaterial desselben handeln.

Transekt 3 Südwest: Der Transekt schneidet etwa $1 \mathrm{~km}$ WSW von T2 SW das bereits dort erfaßte SW-Trockental. Ebenso wie in T2 SW ist es auch in T3 SW asymmetrisch angelegt; die südliche Talflanke fällt steiler ein als die nördliche. Jedoch liegt die zugehörige Talschulter ca. $1 \mathrm{~m}$ höher als die gegenüberliegende. Ähnlich wie in T2 SW korrespondiert auch hier der steilere Hang mit der sohlwärtig angrenzenden Hauptrinne. Somit ist seine Entstehung vermutlich auf ehemalige Prallhangerosion zurückzuführen. Der stratigraphische Aufbau entspricht in groben Zügen dem bereits bekannten: Oberhalb der glazifluvialen Basisschotter folgen fluviale schluffige bis grobsandige Rinnensedimente, die im Unterschied zu T2 SW von einem Seekreidesediment überdeckt werden. Das Profil schließt zum Top mit Niedermoortorfen ab, die je nach Zersetzungsgrad und Schichtfarbe in mehrere Subhorizonte aufgeteilt wurden (im Schichtprofil nicht dargestellt). Insgesamt ist hier die Torflage etwas mächtiger als in T2 SW. Die gestörten Schichtverläufe zwischen X+260 m und X+270 m haben ebenso wie bei T2 SW den Bau des Abwassersammlers als Ursache.

Auf eine Darstellung der Bohrbefunde der übrigen Transekte wird hier verzichtet, da in diesen Fällen im Zuge der Bohrarbeiten häufige Materialverluste auftraten und die Schichtabfolge deshalb nur unvollständig erfaßt werden konnte (vgl. aber die Grubenprofile in Abb. 6).

Da die Absoluttiefen der Hauptrinnen der Transsekte T2 SW und T3 SW mit jeweils etwa $4 \mathrm{~m}$ sehr eng beieinander liegen, die Verbindung beider Rinnensohlen generelles SW-Gefälle anzeigt und auch die Rinnenquerschnittsflächen einander entsprechen, werden sie miteinander verknüpft und zusammenfassend als SW-Rinne bezeichnet. 

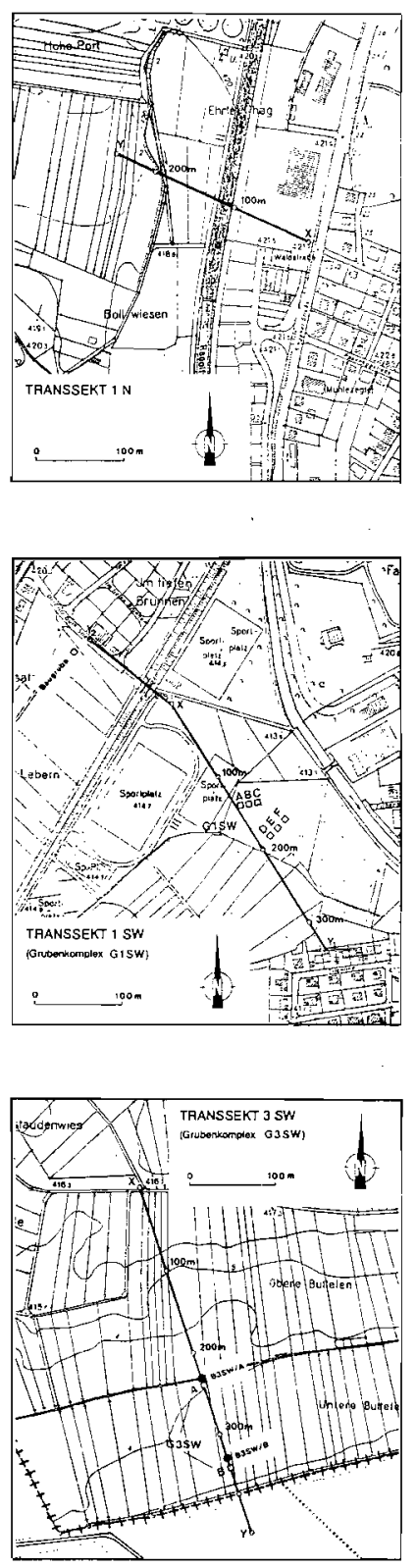
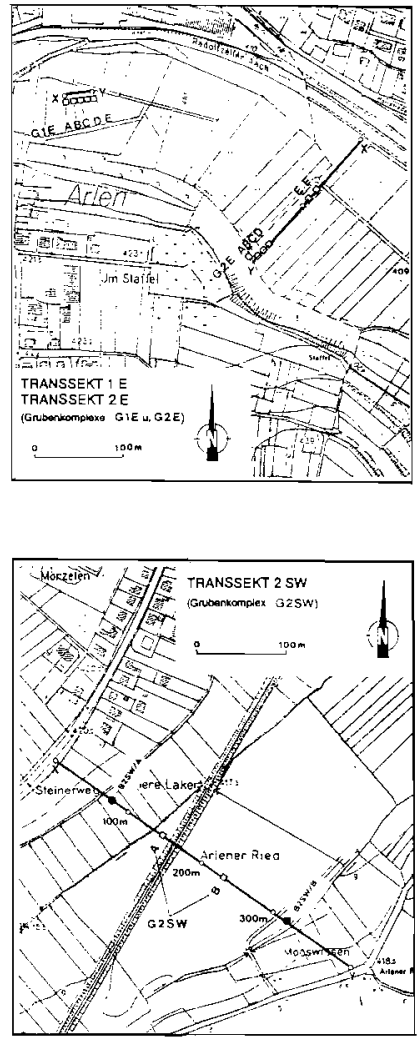

Abb. 4:

Topographische Lage der einzelnen

Bohrtransekte (-), Baggerschürfen ( $\square$ ) und Rammkernbohrungen $(\bullet)$.

(Kartengrundlage: DGK 5). 


\subsection{Lithostratigraphie der Baggerschürfe}

Die Ergebnisse der stratigraphischen und lithologischen Aufnahme der Grubenkomplexe G1SW, G2SW, G3SW, G1E sowie G2E sind in den Abb. 6 a-f dargestellt (Positionsübersicht vgl. Abb. 3 und 4). Die in den Baggerschürfen angetroffenen Ablagerungen wurden wie folgt klassifiziert (vgl. Legende zu Abb. 5 sowie Tab. 1):

- Torfe: rötlich-braune bis schwärzlich-braune, stark humifizierte und in den Toplagen meist stark vererdete Niedermoortorfe mit Wassergehalten der entnahmefrischen Proben von $80-90 \%$ und Glühverlusten von mehr als $80 \%$ der Trockensubstanz (TS). Die Niedermoortorfe sind bei hohem Grundwasserstand in situ aufgewachsen.

- Seekreiden: gelblich-hellgraue Schluffe mit mäßig guter Sortierung, geringem Gehalt an organischer Substanz und hohen Karbonatgehalten. Die Seekreideschichten sind massig ausgebildet und zeigen keine sichtbare texturelle Differenzierung wie Laminierungen, Bänderungen oder Gradierungen. Sie sind ebenfalls in situ entstanden oder nur geringfügig umgelagert (zur Interpretation vgl. Abschn. 5.2).

- Onkolithische Krïmelkalke: hellgraue, meist mittelsandige Sedimente mit charakteristisch geformten grobsandigen bis feinkiesigen Karbonatkonkretionen. Die größeren Konkretionen sind stark verrundet, die kleineren stellen Trümmer und Abrieb der erstgenannten dar. Der Gehalt an organischer Substanz ist gering; der Karbonatgehalt liegt zwischen 350 und $750 \mathrm{~g}^{*} \mathrm{~kg}^{-1}$. Die Krümelkalke werden als fluviale Karbonatausfällungen angesehen, die nach unterschiedlich langem Transportweg an ihre sekundäre Lagerstätte gelangt sind.

- Silikatkiese: Sedimente mit Korngrößenmedianen zwischen $\zeta=0,2 \ldots$ 1,32. Die Korngrößenverteilung verläuft stets rechtssteil mit scharfem Abfall der Klassenhäufigkeit beim Übergang Grobkies/Steine; mitunter treten sekundäre Häufigkeitsmaxima im Sand- und Schluffbereich auf. Es handelt sich um ältere Glazifluvialsedimente, die bei starker Wasserführung transportiert wurden.

- Silikatische Sande und Schluffe: Der markanteste Unterschied zu den obigen Silikatkiesen liegt in dem (abgesehen von einer Ausnahme) durchwegs linkssteilen Verlauf der Korngrößenverteilungen. D.h. in Bezug auf den Median sind die feinerkörnigen Anteile stets schwächer vertreten als die gröberen. Bei den Sanden treten gelegentlich bimodale Verteilungen auf. Dies läßt darauf schließen, daß in diesen Fällen heterogene Transportbedingungen (hohe Variabilität der Fließgeschwindigkeit) gegeben waren. Auch an selektiven Materialtransport (z.B. nachträgliche Materialein- 


\begin{tabular}{|c|c|c|c|c|c|}
\hline Probe (LE) & $\begin{array}{c}\mathbf{K}^{+} \\
\left|\mathrm{g}^{\star} \mathrm{kg}^{-1}\right|\end{array}$ & $\begin{array}{l}X_{\mathbf{M g}_{\mathbf{g}}} \\
{\left[\%_{0}\right]}\end{array}$ & $\begin{array}{l}\mathrm{X}_{\mathrm{Sr}} \\
{[\% \text { ] }}\end{array}$ & $\begin{array}{c}\text { Ges.-Karhonat } \\
\qquad\left[g^{\star} \mathrm{kg}^{-1}\right]\end{array}$ & $\begin{array}{c}\text { os } \\
{\left[g^{*} \mathrm{~kg}^{-1}\right]}\end{array}$ \\
\hline GISW/B-2.1 & 0.96 & 18.9 & 1.21 & 401 & 201 \\
\hline GISW/E-2 & 0.39 & 8.7 & 0.31 & 740 & 35 \\
\hline GlE/C-3 & 0.38 & 25.5 & 0.59 & 332 & 37 \\
\hline G2E/A-5 & 0.30 & 13.1 & 0.32 & 651 & 35 \\
\hline G2SW/A-2 & 0,63 & 99.0 & 0.76 & 175 & 108 \\
\hline G2SW/A-3 & 0.45 & 68.3 & 0.70 & 345 & 22 \\
\hline G3SW/A-2.I & 0.02 & 28.1 & 0.60 & 936 & .36 \\
\hline
\end{tabular}

Tab. 1: Ergebnisse der geochemischen Untersuchungen ausgewählter Proben. (OS: Organische Substanz; $\mathrm{X}_{\mathrm{Mg}}, \mathrm{X}_{\mathrm{Sr}}$ : Mg- bzw. Sr-Molenbruch. Beisp. $\mathrm{X}_{\mathrm{Mg}}:=\left(\left[\mathrm{Mg}^{2+}\right]^{*} 1000\right) /\left(\left[\mathrm{Ca}^{2+}\right]+\left[\mathrm{Mg}^{2+}\right]+\left[\mathrm{Sr}^{2+}\right]\right)$.

schwemmungen aus dem Hangenden) kann gedacht werden. Die Schluffe allerdings weisen in fast allen Fällen eine recht gute Korngrößensortierung verbunden mit hohen Glühverlustwerten auf. Da sowohl die Sande als auch die Schluffe stets im Bereich der in die Schotterbasis eingeschnittenen Rinnen angetroffen wurden, werden sie im Vergleich zu den Glazifluvialkiesen als jüngere Fluvialsedimente angesprochen.

G1SW (Abb. 6 a und b): Die Grubensequenzen $A$ bis $C$ sowie $D$ bis $F$ zeigen jeweils eine an zwei verschiedenen Stellen angeschnittene Rinne, die bereits durch ihre Oberflächenmorphologie aufgefallen war. Die Lithostratigraphie bestätigt den äußeren Befund. Während in den Randgruben, abgesehen vom geringmächtigen Oberbodenhorizont, ausschließlich Grobsedimente anstehen, liegen im Rinnenzentrum teilweise feinklastische Schichten mit hohem organischem Anteil. Kurzkerne wurden hier nicht entnommen. Aus G1SW/B-4 liegt eine ${ }^{14} \mathrm{C}$-Datierung vor.

G1E (Abb. 6c): Die Gruben A, D und E liegen eher rinnenperipher und sind wie die Randgruben aus G1SW aus teilweise differenzierten Kieslagen mit aufliegendem Oberboden aufgebaut. Die um etwa $0,5 \mathrm{~m}$ tiefer liegenden Zentralgruben $\mathrm{B}$ und $\mathrm{C}$ enthalten feinsedimentäre Schichten mit vereinzelt hohem Glühverlust (z.B. B-3, vgl. Tab. 1). Auch größere Holzreste kommen gehäuft vor. Die Basis der Rinne wird von einem homogenen Karbonatsand überlagert ( $\mathrm{B}-4 ; \mathrm{C}-3, \mathrm{vgl}$. Tab. 1).

G2E (Abb. 6 d): Die Gruben A und B weisen bis zu $1 \mathrm{~m}$ mächtige Torfschichten auf. In Grube A wird der Torf von stark humosem Lehm unter- 


\section{T1 $\mathrm{N}$ (a)}

Höhe u. NN [m]

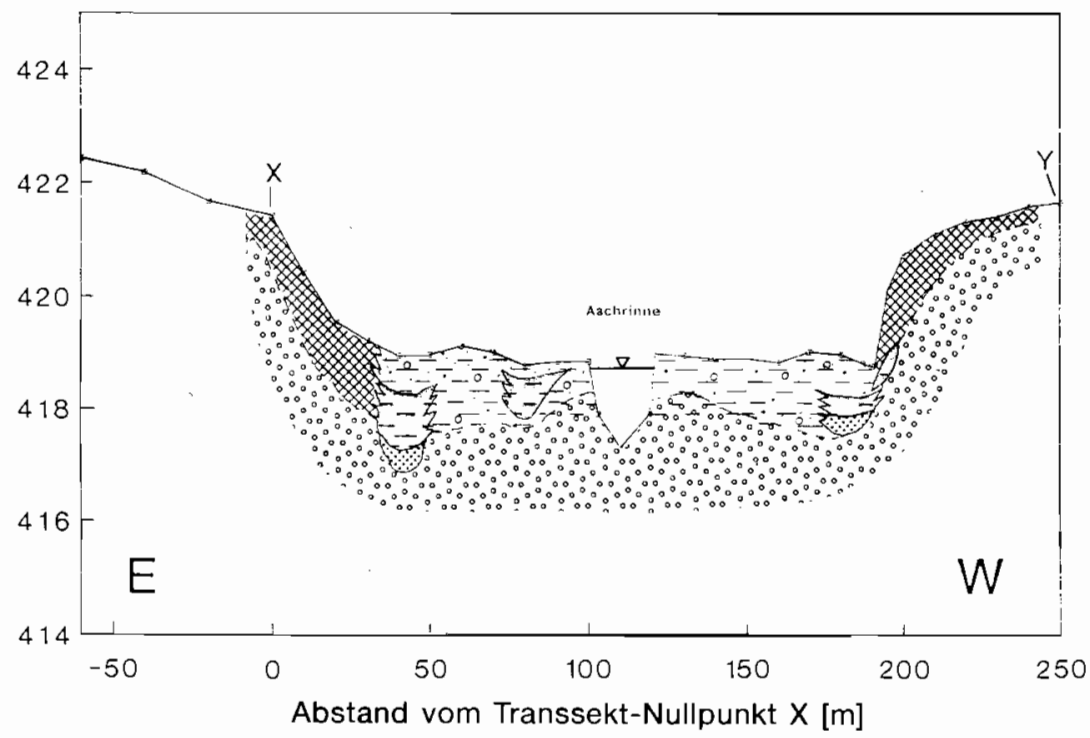

LEGENDE (Gruben- u. Bohrprofile)

Organogene Sedimente (u. Oberboden)

Silikatische Mischsedimente
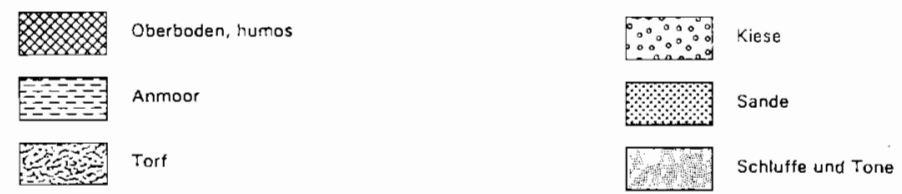

Karbonatsedimente

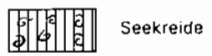

Sonstige

$\Delta_{\Delta}^{\Delta \Delta \Delta \Delta^{\Delta} \Delta \Delta^{\Delta} \Delta^{2}}$ Anthropogene Aufschüttungen

गागा|| Onkolithische Krümelkalke, homogen

$\because[0.010$ Onkolithische Krümelkalke, heterogen

Abb. 5: Lithostratigraphische Querprofile der Bohrtransekte T 1N (a), T 2SW (b) und T 3SW (c). (stark überhöht). 


\section{T2 SW (b)}

Höhe i. NN [m]

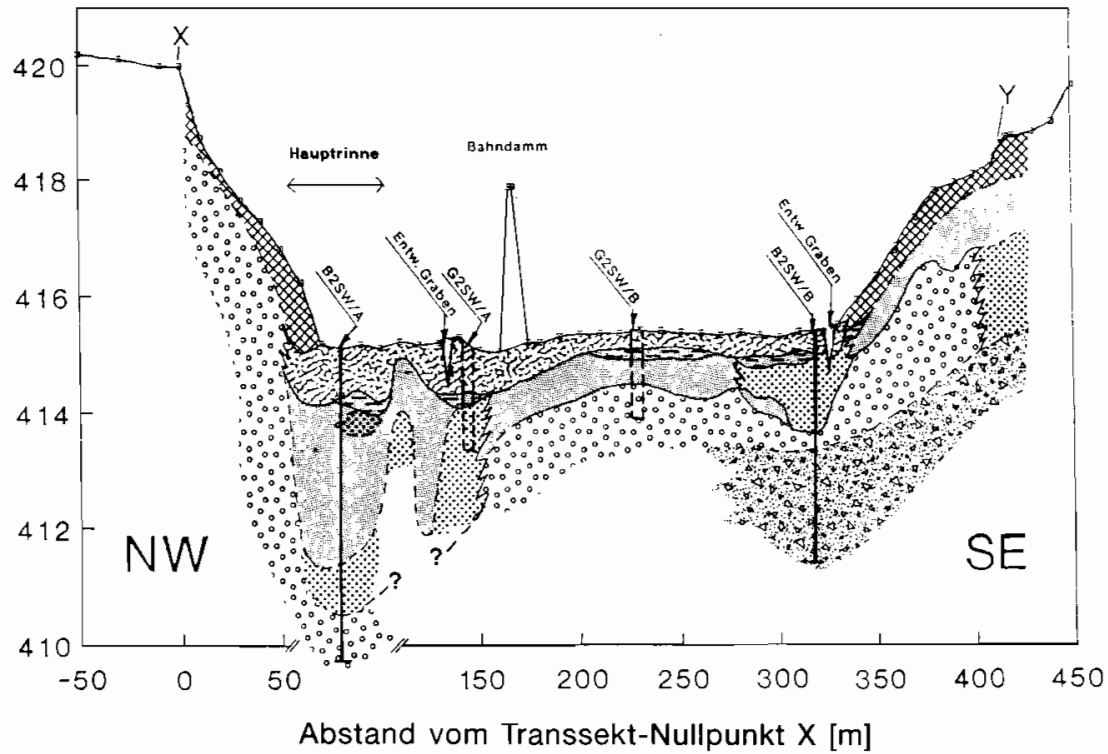

T3 SW (c)

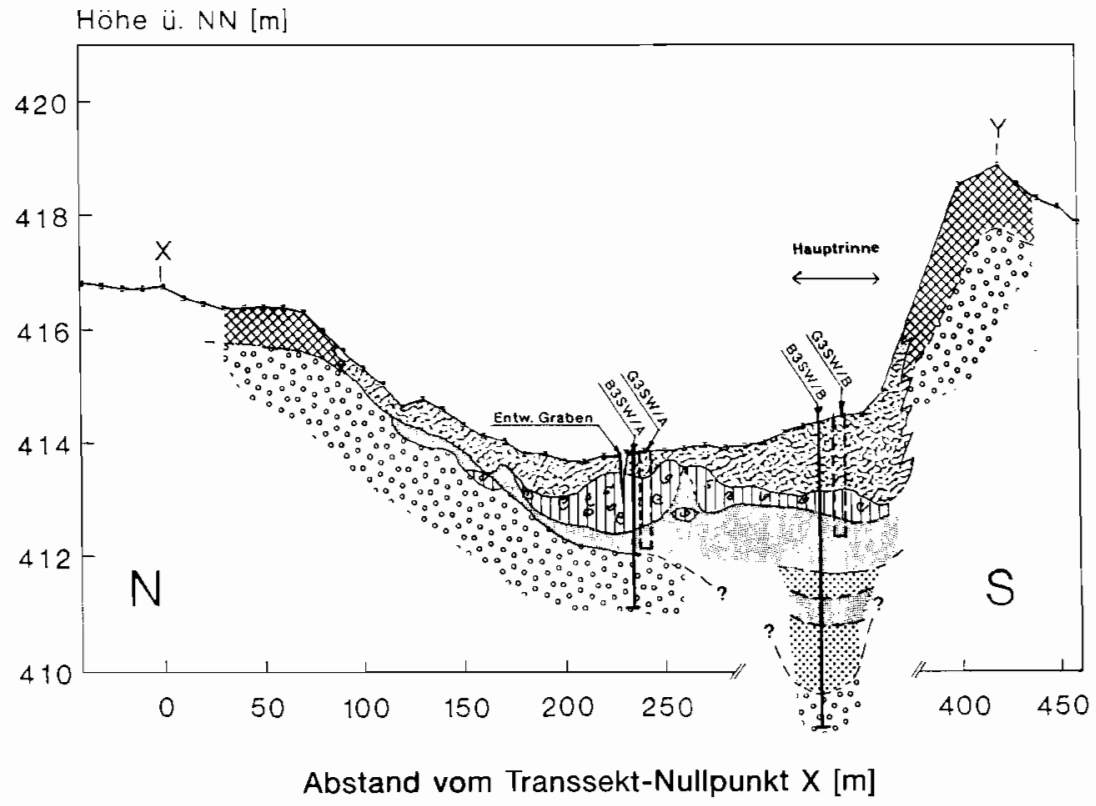



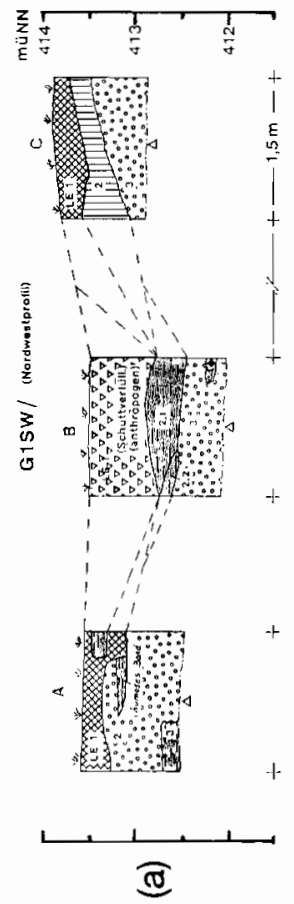
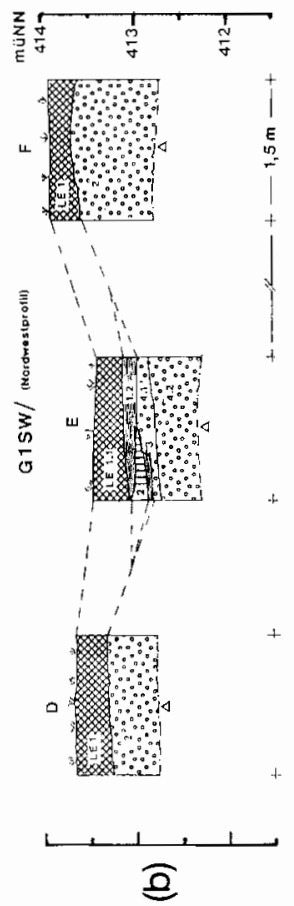
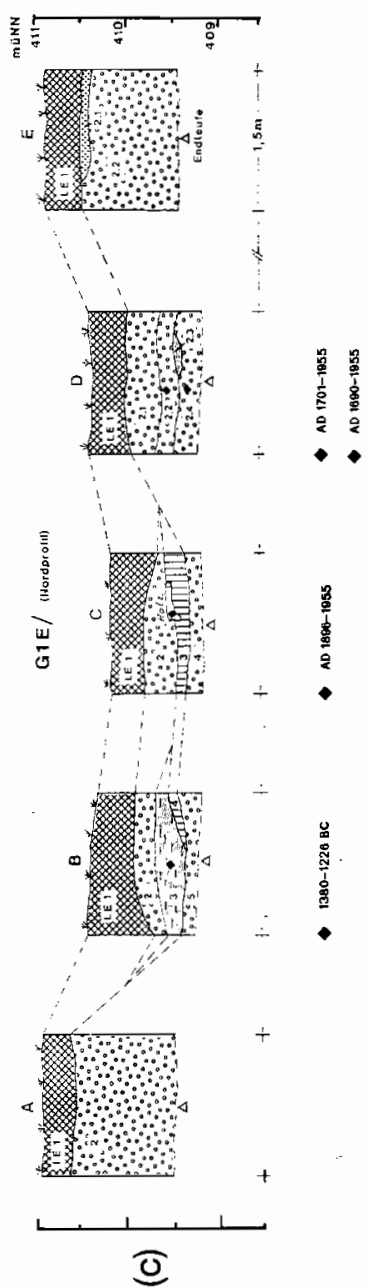

Abb. 6: Lithostratigraphische Querprofile der Baggerschürfe. G1SW (a, b), G1E (c), G2E (d), G2SW (e) und G3SW (f). 

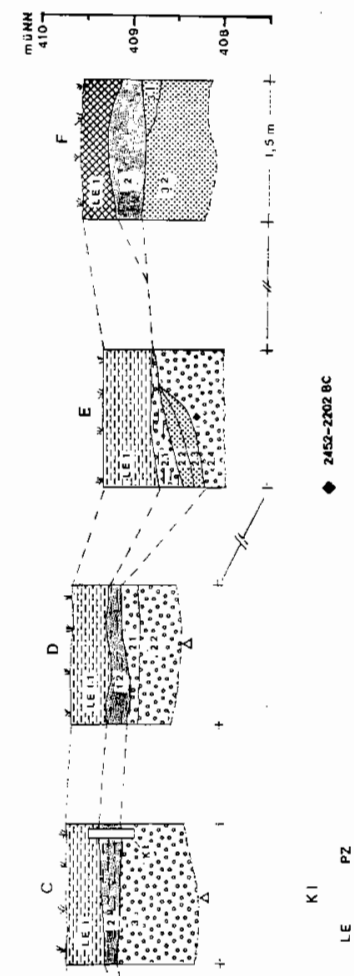

m
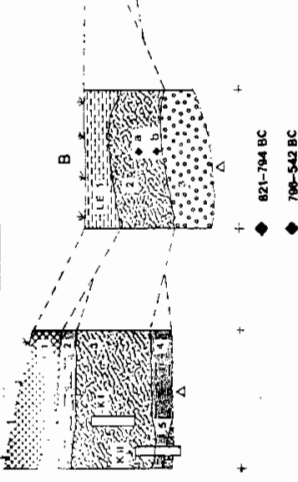

흐
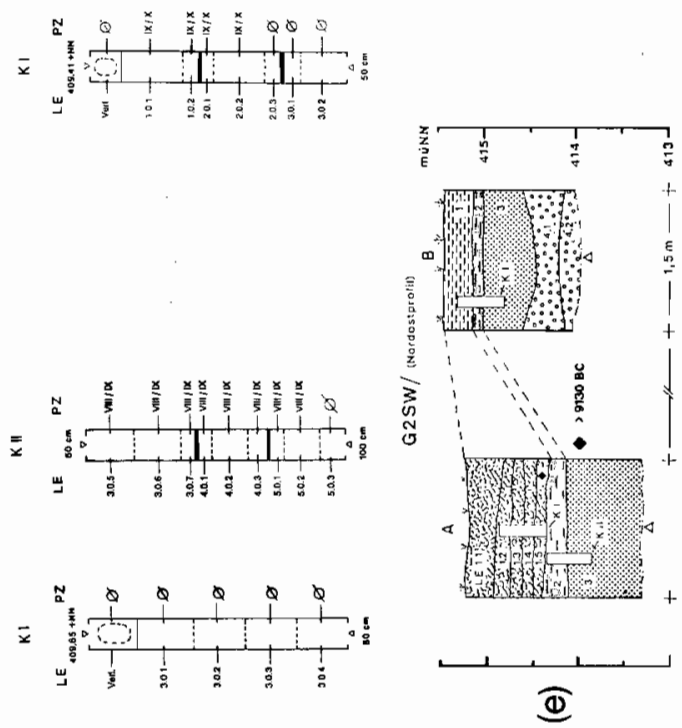
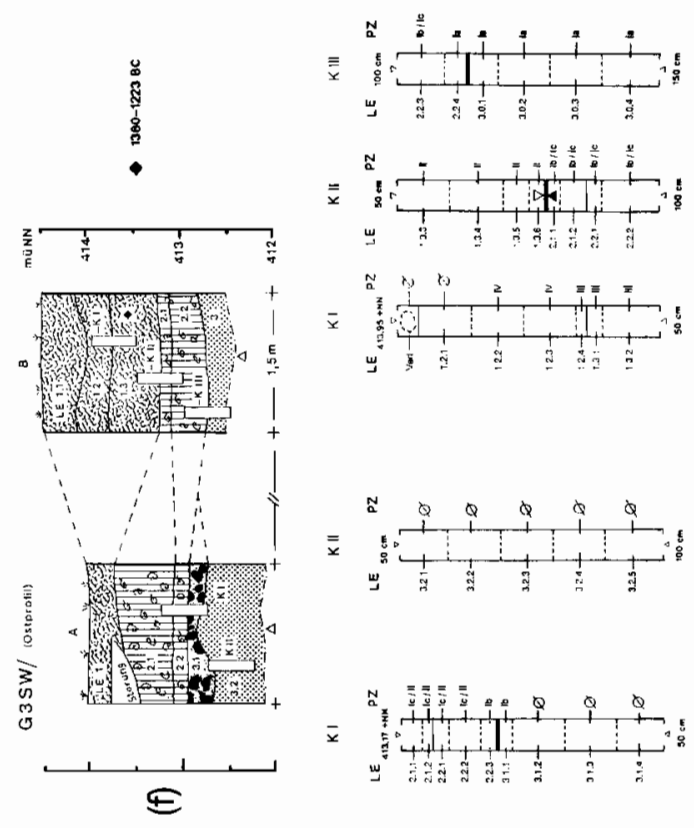
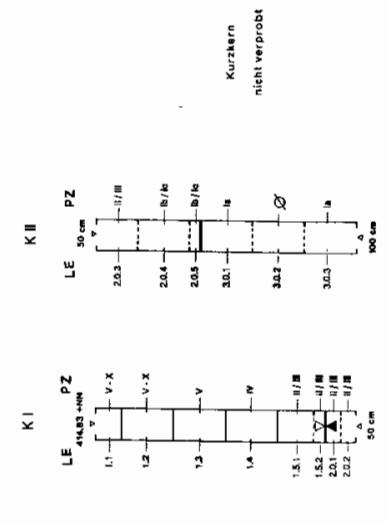
lagert, der wiederum einer reinen, mittelkörnigen Karbonatlage (A-5) aufliegt. Hier scheint eine von Verlandungssedimenten überdeckte Rinne gelegen zu haben. Die Gruben C und D liegen peripher und zeigen die "Standardabfolge" Oberboden - Kies. Allerdings sind hier geringmächtige Lehmlagen mit hohem Glühverlust zwischengeschaltet. Diese werden als Auelehm angesprochen. Die Grube E zeigt eine in die horizontal lagernde Kiesbasis eingelassene Abfolge von drei deutlich nach SW einfallenden Einzelschichten. Somit ist anzunehmen, daß hier der Flankenbereich einer weiteren verlandeten Rinne lokalisiert ist. Grube F liegt bereits außerhalb dieses Rinnenbereiches und weist einen ähnlichen Schichtbau wie die Gruben $\mathrm{C}$ und $\mathrm{D}$ auf.

G2SW und G3SW (Abb. 6 e und f): Die vier Schürfe dieser Grubenkomplexe liegen in den durch Handbohrung sondierten und bereits weiter oben vorgestellten Profilen von T2 SW und T3 SW, so daß hier auf eine zusätzliche Besprechung der Stratigraphie verzichtet werden kann. Als Besonderheit sei aber auf das „Steinpflaster“ (G3SW/A-3.1) hingewiesen. Es handelt sich hierbei um eine etwa $20 \mathrm{~cm}$ mächtige Schicht aus kompakt gelagerten und teilweise scharfkantig zerbrochenen Grobkiesen und Steinen mit bis zu $20 \mathrm{~cm}$ Einzeldurchmesser, die von fluvialem Feinmaterial unterlagert und von Seekreide überdeckt ist.

\subsection{Radiokarbon- und Pollendatierung}

Die Ergebnisse der ${ }^{14} \mathrm{C}$-Datierungen sind in der Tab. 2 dargestellt. Die Einzelproben stammen aus 9 unterschiedlichen lithostratigraphischen Einheiten von insgesamt 8 verschiedenen Baggergruben. Bei Probe G2E/E-2.4 war der Erhaltungszustand des Holzes derart schlecht, daß nicht ausgeschlossen werden kann, daß es sich hierbei um Wurzelmaterial handelt, das nicht unbedingt schichtdatierend ist. Probe G2SW/A-1.5 (Torfbasis) lag altersmäßig im Grenzbereich des derzeitig aktuellen Kalibrationsprogrammes. Ihr siderisches Höchstalter beträgt laut mdl. Mitt. d. Inst. f. Umweltphysik, Heidelberg, 9800 BC.

Die entnommenen Pollenproben dienen der Datierung der Stratigraphien und nicht vegetationsgeschichtlichen Rekonstruktionen. Daher sind die Pollenprofile in vereinfachter Form dargestellt (Abb. 7 a-e). Die Pollenerhaltung war insbesondere bei den Proben des Profils G2E/A-KI sehr schlecht, aber auch bei anderen Profilen besteht der Verdacht auf selektiven Pollenzersatz, da oft ungewöhnlich hohe Tilia-Werte auftraten.

Profilsäulen G2E/A-KI und -KII (Datierung: Subboreal/Subatlantikum, VIII/IX; Abb. 7 a): Nur die LE 5.0.2 bis 3.0.5 waren pollenführend. Die überlieferte Vegetationsentwicklung beginnt mit einem Laubmisch- 


\begin{tabular}{|c|c|c|c|c|}
\hline $\begin{array}{l}\text { Probe (LE) } \\
\text { (Labomummer) }\end{array}$ & Holzart & Bereich & ${ }^{14} \mathrm{C}$-Alter (BP) & ${ }^{14}$ C-Alter (cal.) \\
\hline $\begin{array}{l}\text { Gisw/B-4 } \\
\text { (HD 15530-14910) }\end{array}$ & Rinde, unbek. & Rindenholz & $5315 \pm 50$ & $\$ 236-1041 \mathrm{BC}$ \\
\hline $\begin{array}{l}\text { GlE/B-3 } \\
\text { (HD 15587-14906) }\end{array}$ & Jirburnum sp. & Stammholz & $3020 \pm 35$ & $1380-1228 \mathrm{BC}$ \\
\hline $\begin{array}{l}\text { GIE/C-2 } \\
\text { (HD 15379-14226) }\end{array}$ & Pinus sp. & Stamın-Bretı & $55 \pm 35$ & $A D 1896-1955$ \\
\hline $\begin{array}{l}\text { GlE/D-2 } 2 \\
(\mathrm{HD} 15376-14866)\end{array}$ & Quercussp & Stammholy & $75 \pm 25$ & $A D 1701 \cdot 1955$ \\
\hline $\begin{array}{l}\text { GIE/D-2 } \\
\text { (HD 15375-15129) }\end{array}$ & Piceasp. & Stanunholz & $95 \pm 20$ & AD $1690-1955$ \\
\hline $\begin{array}{l}\text { G2E/B-2 } \\
\text { (HD 15381-14848) }\end{array}$ & Alnus sp. & Stammholz & $2637 \pm 49$ & $821-794 \mathrm{BC}$ \\
\hline $\begin{array}{l}\text { G2E/B-2 (Torfbasis) } \\
\text { HD (15377-14856) }\end{array}$ & Fagus sp. & Stammhol/. & $2520 \pm 50$ & $796-542 \mathrm{BC}$ \\
\hline $\begin{array}{l}\text { G2E/E-2.4 } \\
\text { (HD 15382-14911) }\end{array}$ & Almus sp. & est Wureel & $3830 \pm 45$ & $2452-2202 \mathrm{BC}$ \\
\hline $\begin{array}{l}\text { G2SW/A-1.5 } \\
\text { (Torlbasis) } \\
\text { (HD 15378-14903) }\end{array}$ & Pinus sp. & Stammhole & $10040 \pm 50$ & $>9130 \mathrm{BC}$ \\
\hline $\begin{array}{l}\text { G.3SW/B-1.3 } \\
\text { (HD 15380-14845) }\end{array}$ & Alnus sp. & Stammholz & $3015 \pm 40$ & $1380 \cdot 1223 \mathrm{BC}$ \\
\hline
\end{tabular}

Tab. 2: Ergebnisse der ${ }^{14} \mathrm{C}$-Altersbestimmungen.

wald mit Fagus, Quercus, Tilia und Corylus. Dominiert wird die Pollenflora aber von Alnus-Pollen, die teilweise mehr als $50 \%$ der erfaßten Gesamtpollensumme (GPS) ausmachen. Ein kurzer Vorstoß von Pinus, der mit einem kurzen Rückzug von Alnus einhergeht, beeinträchtigt mit Ausnahme von Tilia den Laubmischwald kaum. Die hohen Linden-Anteile können angesichts der Tatsache, daß Ulmus nur noch in Spuren vorhanden ist, nicht dem Atlantikum entsprechen, sondern müssen sekundär zur Dominanz gekommen sein; auch der sehr schlechte Erhaltungszustand fast aller übrigen Pollentypen spricht dafür. Die Datierung stützt sich im wesentlichen auf die Fagus-Kurve. Die Anteile erreichen etwa $10 \%$ der GPS. Dies würde zumindest den Beginn des Subboreals bedeuten (LANG 1982, Rösch 1990 a). Zieht man aber in Betracht, daß die Werte von Tilia eigentlich unter $1 \%$ liegen sollten, alle anderen Prozentanteile dementsprechend erhöht wer- 


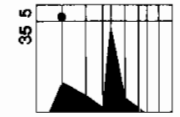

Polypodium vulgaris

Polypodiaceae
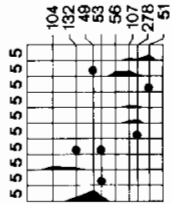

Gesamtsumme

Typha angustifolia

Filipendula

Brassicaceae

Ranunculaceae

Apiaceae

Cichoriaceae

Chenopodiaceae

Thalictrum

A

옹ํㅇํํ음워

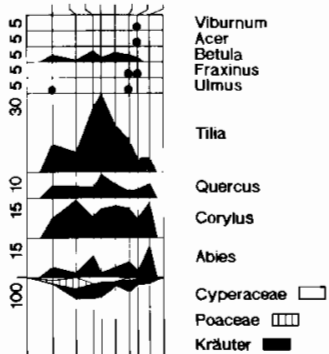

世政

8
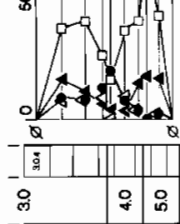

Alnus $\square$

Fagus $\boldsymbol{A}$

Pinus

polientrei $Q$

Lithostratigraphie ILE]

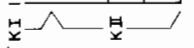

先高

Tiefe $1 \mathrm{~cm}$ ab OK-Kern I $[0=409,65 \mathrm{mNN}]$

(a)
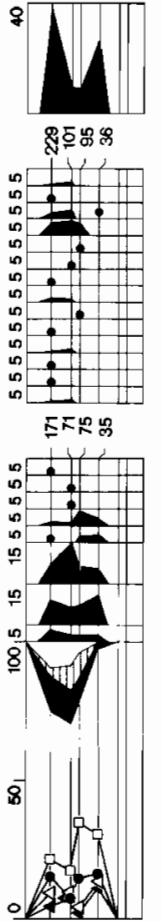

Fagus

Pinus

Picea $\triangle$

pollenfrei $Q$

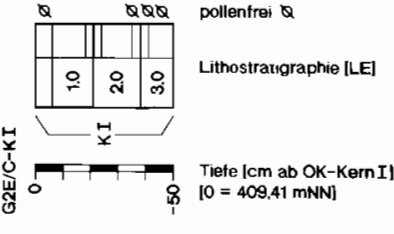

(b)

Cyperaceae $\square$

Poaceae एس

Kràuter

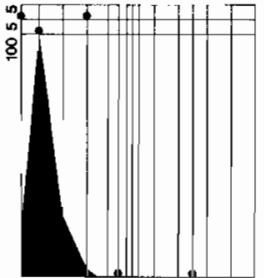

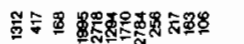

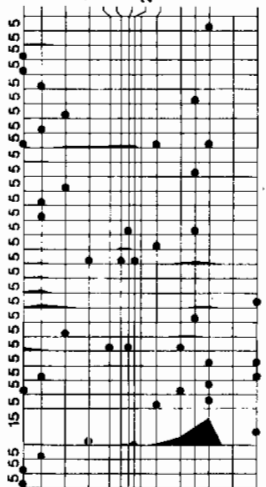

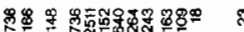

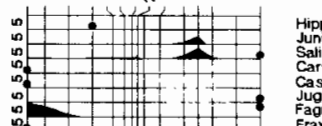

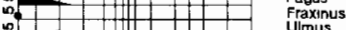

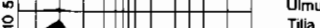

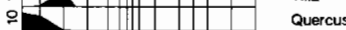

8
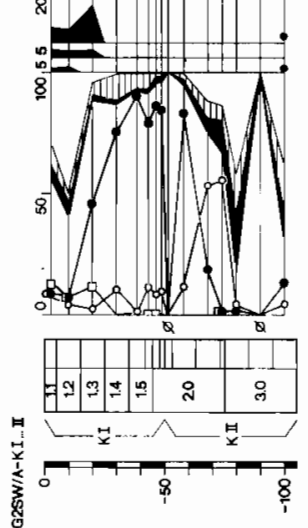

Antula

Pinus

pollentrei $Q$

Lithos tratıraphie [LE]

Tiete $\mathrm{Icm}$ ab $O K-K e r n I$ $10=414,83 \mathrm{mNN}]$

(c)

Abb. 7: Pollendiagramme der untersuchten Kurzkerne. G2E/A-KI-II (a), G2E/ C-KI (b), G2SW/A-KI-II (c), G3SW/A-KI (d), G3SW/B-KI-III (e). 


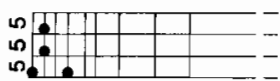

Sphagnum

Botrychium

Selaginella

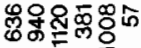

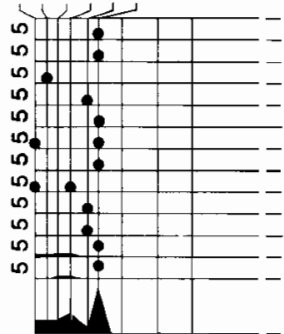

항용요요요
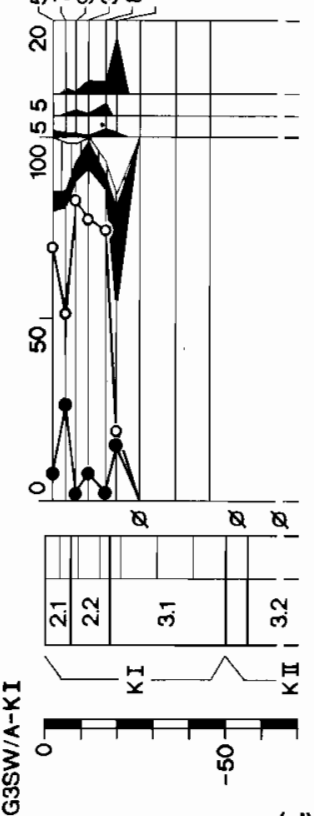

Betula o

Pinus

pollentrei $Q$

Lithostratigraphie [LE]

Tiefe [cm ab OK-Kern 1$]$ $[\mathrm{O}=413,17 \mathrm{mNN}]$

(d)

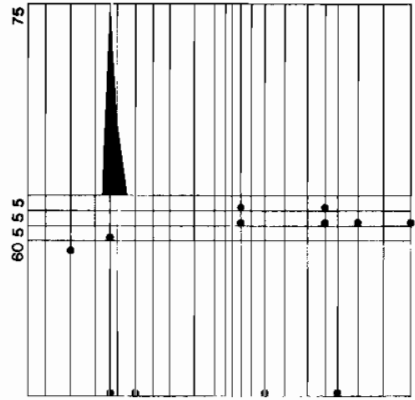

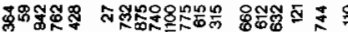

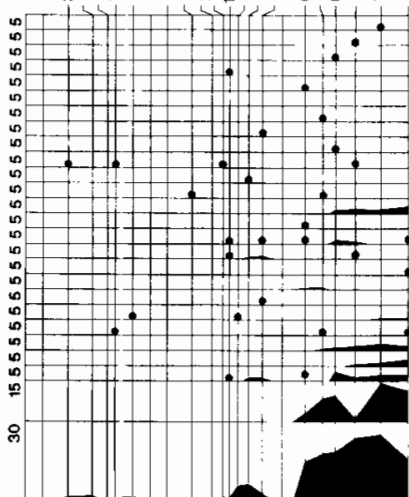

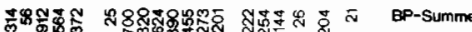

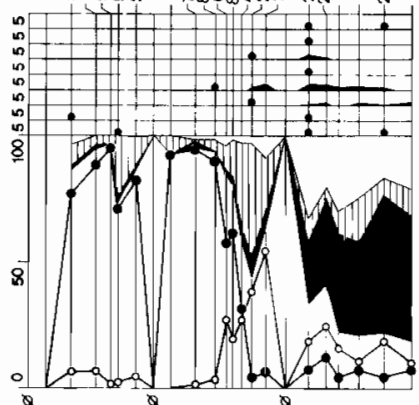

Ephedra dislachya

Calluna

Hippophae

Juniperus

Saix
Alnus

Picea

Cyperaceae $\square$

Poaceae [II

Kräuter $\square$

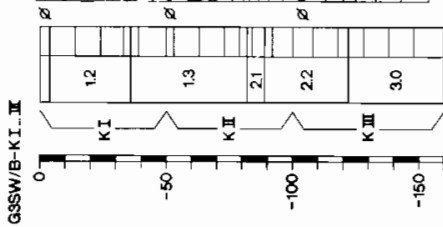

pollentrei $Q$

Lithostratigraphie [LE]

(e) 
den müßten, wird eine Einstufung ins Subatlantikum als am wahrscheinlichsten angesehen. Dem widersprechen auch nicht die fehlenden Siedlungszeiger, die vermutlich völlig zerstört worden sind.

Profilsäule G2E/C-KI (Datierung: Subatlantikum, IX/X; Abb. 7 b): Ähnlich wie in den obigen Profilsäulen ist die Pollenerhaltung sehr schlecht. Die relativ hohen NBP-Prozentanteile, verbunden mit Spuren von Kulturzeigern (Triticum, Secale), lassen dieses Profil in den jüngeren Teilen etwas vollständiger erscheinen als das vorige. Hingegen ist der Übergang Subboreal/Subatlantikum wahrscheinlich in den pollenfreien Abschnitten LE 2.0.3 bis 3.0.2 zu suchen.

Profilsäulen G2SW/A-KI und -KII (Datierung: Älteste Tundrenzeit bis Boreal, Ia bis V, am Top jüngstes Holozän; Abb. 7 c): Wegen des großen Probenabstandes ist nur eine grobe Einteilung der Sedimente möglich. Der Abschnitt zwischen LE 3.0.3 und 3.0.1 stellt pollenarme Ablagerungen dar, in denen neben Steppenelementen wie Artemisia und Thalictrum Poaceen und Cyperaceen dominieren. Dies entspricht den Untersuchungsergebnissen von LANG (1982) am Mindelsee, der dort ebenfalls eine sehr lokal geprägte, feuchtigkeitsliebende Flora der Ältesten Tundrenzeit nachweisen konnte. Die weitere Entwicklung des Spätglazials ist in groben Zügen vollständig erhalten. Das Bølling (LE 2.0.5 und 2.0.4) macht sich durch einen kräftigen Birkenvorstoß bemerkbar, während die Jüngere Tundrenzeit fast gar nicht angedeutet ist. Allerød, Jüngere Tundrenzeit, Präboreal und Boreal können zwischen LE 2.0.3 und 1.3 nur andeutungsweise ausgegliedert werden. Oberhalb von LE 1.3 sind dann wahrscheinlich nur die jüngsten Teile des Holozäns überliefert (Fagus, Carpinus und Spuren von Kulturzeigern sind bereits vorhanden). Aufgrund des großen Probenabstandes kann nicht entschieden werden, ob ein größerer Hiatus oder ein stark komprimiertes Profil vorliegt, wie es im Bereich von Flußauen häufig vorkommt (z.B. Torfsackung o.ä., vgl. KoRTFUnKE 1992).

Profilsäulen G3SW/A-KI und -KII (Datierung : Bølling/Allerød, Ib/II; Abb. 7 d): Zwischen LE 3.2.5 und 3.1.2 ist das Profil pollenfrei. Die LE 3.1.1 und 2.2.3 lassen sich wegen der hohen Betula-Anteile dem Bølling zuordnen. Inwieweit die Schwankungen von Pinus und Betula in den LE 2.2.2 bis 2.1.1 schon zur Älteren Tundrenzeit oder gar zum Allerød gehören, läßt sich aus der kurzen Sequenz nur schwer ableiten.

Profilsäulen G3SW/B-KI bis KIII (Datierung: Älteste Tundrenzeit bis Präboreal, Ia bis IV; Abb. 7 e): Das Profil umfaßt zwischen den LE 3.0.4 und 1.2.2 eine recht vollständige Abfolge des Spätglazials, beginnend mit einer sehr deutlich ausgeprägten Ältesten Tundrenzeit (LE 3.0.4 - 2.2.4) 
mit sehr hohen Anteilen von Artemisia (um $20 \%$ ) und Helianthemum (bis $15 \%$ ). Poaceen und Cyperaceen spielen (im Gegensatz zum Profil G2SW/A-KII) nur eine geringe Rolle. Das Bølling macht sich wiederum durch einen kräftigen Birkenvorstoß bemerkbar, das Allerød durch die Ausbreitung von Pinus. Die Jüngere Tundrenzeit ist nur in Andeutungen vorhanden (LE 1.3.2 - 1.2.4). Auch hier muß auf den großen Probenabstand verwiesen werden, der eine weitergehende zeitliche Auflösung nicht zuläßt.

\subsection{Geochemie, Partikelanalyse und Mollusken}

Die Ergebnisse der geochemischen Untersuchungen, der Partikelzählungen sowie der Bestimmung von Wirbellosenresten sind in den Tab. 1,3 und 4 dargestellt.

Die Probe G1SW/B-2.1 enthält hohe Quarzit-, Silikat- und Kalkgesteinsanteile, die vermutlich aus der Gewässersohle stammen. Weiterhin sind Partikel reichlich vertreten, die im Flußbett entstanden und transportiert worden sind, v.a. rundliche Karbonatkrusten, Wassermolluskenreste (s.und) und vereinzelte Köcherreste von Köcherfliegen-Larven (Trichoptera, Insecta). Die Karbonatkrusten sind allseitig abgeschliffen, verraten also fluvialen Transport. In ihrer äußeren Form sowie vom inneren Aufbau her ähneln sie zerfallenen Schneggli-Kiesen, wie sie vom Bodensee-Ufer bekannt sind. Die hohe Abundanz von Ostracoden-Schalen läßt auf Stillwasserbedingungen zumindest an der Gewässersohle schließen. Zu den eher terrestrischen Partikeln, die aus den Uferbereichen in das Flußbett eingetragen wurden, gehört der reichlich vertretene Grobdetritus, die Mehrzahl der Samen und Früchte, die Chitin-Teile (meist Flügeldeckenund Halsschildreste von Käfern) sowie vereinzelte Fliegen-Puparien und Moosmilben.

In der Probe wurde eine verhältnismäßig artenreiche, aber individuenarme Land- und Wasserschneckenfauna angetroffen. Der Landschneckenanteil mit den dominanten Arten Carychium minimum und Vallonia pulchella ist geringfügig höher als der Wasserschneckenanteil, in dem die Arten Radix ovata, Planorbis carinatus, Gyraulus aeronicus und Hippeutis complanatus, welche für pflanzenreiche stehende Gewässer kennzeichnend sind, überwiegen. Die genannten Landschnecken leben in offenen sumpfigen Biotopen, und auch die gefundenen Insektenreste stammen von Formen aus dem wechselfeuchten bzw. submersen Uferbereich.

Bei der Probe G1SW/B-2.1 handelt es sich vermutlich um zusammengespülte Reste aus dem Flußbett sowie aus benachbarten terrestrischen Bereichen, die sich infolge abnehmender Transportkraft des Wassers hier ab 


\begin{tabular}{|c|c|c|c|}
\hline Probe (LE) & G1SW/B-2.1 & $\begin{array}{c}\text { G2SW/A-2 } \\
\text { (untere Hălfte) }\end{array}$ & $\begin{array}{c}\text { G3SW/A-2.1 } \\
\text { (obere Hälfte) }\end{array}$ \\
\hline Sediment-Typ & Schwemmtorf & humoser Lehm & Seekreide \\
\hline Pollendatierung & n.b. & $\begin{array}{c}\text { Jüngere } \\
\text { Tundrenzeit }\end{array}$ & Bolling-Allerod \\
\hline${ }^{14}$ C-Alter (cal.) & $<4236-4041 \mathrm{BC}$ & $>9130 \mathrm{BC}$ & n.b. \\
\hline Quarzite & 3.81 & 3,95 & 0,13 \\
\hline Silikatgesteine & 3,64 & 4,42 & 0,66 \\
\hline Kalkgesteine & 2.55 & 2,90 & 0,21 \\
\hline unregelmäßig-kugcligc Karbonatkrusten & 3.98 & n.n & n.n \\
\hline Characeen-Krusten & ก. n & п.п & 4,74 \\
\hline sonstige Karbonatkrusicn & -0.24 & ח. ח & n.n \\
\hline Holzkohlen & 1.52 & 1.82 & n. $n$ \\
\hline Grobdetritus (unverkohlt) & 4.51 & 5.04 & 3,69 \\
\hline Grobdetritus (verkohll) & -0.06 & 1.52 & n.n \\
\hline Samen u. Früchte (unverkohlt) & 2.70 & 2.00 & 0,97 \\
\hline sonstiger Strukturdetritus (unverkohlt) & 1.52 & 2.12 & ก.n \\
\hline Characeen-Oogonien u -Oosporen *) & ก. $n$ & 5.06 & 3,44 \\
\hline Molluskenschalen (vollst. erhalten) & 1.98 & 3,91 & 2.92 \\
\hline Mollusken-Trümmcrschill & 3.78 & 4.33 & 3.46 \\
\hline Bithynia-Deckel & 2.10 & n.n & n.n \\
\hline $\begin{array}{l}\text { Chitin-Teile (Flügeldecken. Halsschilder. } \\
\text { Beintcile etc.) }\end{array}$ & 2.12 & 2.00 & n. $\mathbf{n}$ \\
\hline unstrukturierte Chitinreste (Eihüllen etc.) & 3,76 & 1.82 & ก.n \\
\hline Flicgen-Puparien & 1.49 & n.n & ก.n \\
\hline Knochensplitter & -0.54 & n.n & n.n \\
\hline Ostracoden-Schalen *) & 4,28 & n.n & 4,35 \\
\hline Trichopteren-Köcherreste & 1.79 & 2.30 & n.n \\
\hline Moosmilben & 2.84 & 2,68 & п.n \\
\hline
\end{tabular}

Tab. 3: Ergebnisse der Partikelanalyse ausgewählter Proben. Dargestellt sind die Partikelgehalte in pn (pn := lg n; n: Partikelanzahl pro kg TS). Untersucht wurde die Fraktion von 0,63 bis $6,3 \mathrm{~mm}$ (Grobsand + Feinkies). Bei den mit "*" markierten Partikelklassen wurde zusätzlich die Mittelsandfraktion $(0,20$ bis $0,63 \mathrm{~mm})$ miterfaßt.

gesetzt haben. Zu denken wäre an Ablagerungen in einer flachen, verlandenden Rinne mit submersem Pflanzenbewuchs. Aus diesen Gründen wird das Sediment hier als „Schwemmtorf" angesprochen.

Der humose Lehm G2SW/A-2 weist eine ähnliche partikuläre Zusammensetzung auf; auch er enthält autochthone Anteile (Wassermollusken, s.und, Trichopteren-Köcherreste, jedoch keine Karbonatkrusten) sowie terrestrische Partikel (Grobdetritus, Strukturdetritus, Chitin-Teile, Moosmilben). Die hohe Abundanz von Characeen-Oogonien deutet auf Stillwasserbedingungen hin, die vermutlich gegeben waren, nachdem sich das übrige Material abgelagert hatte. 


\begin{tabular}{|c|c|c|c|}
\hline Probe (LE) & G1SW/B-2.1 & $\begin{array}{c}\text { G2SW/A-2 } \\
\text { (untere Hălfte) }\end{array}$ & $\begin{array}{c}\text { G3SW/A-2.1 } \\
\text { (obere Hălfte) }\end{array}$ \\
\hline Sediment-Typ & Schwesnmtorf & bumoser Lebm & Seekreide \\
\hline \multicolumn{4}{|l|}{ I. Mollusken (insges.): } \\
\hline GesamLancahl der Molluskenarten & 11 & 7 & 7 \\
\hline $\begin{array}{l}\text { Individuendichte } \\
\text { [Indiv./kg TS] }\end{array}$ & 251 & 5435 & 633 \\
\hline \multicolumn{4}{|l|}{ Ia) Wassermollusticn : } \\
\hline I alvata cristata O.F Müller & n.n & n.n & 40 \\
\hline I ahata piscinalis puc. O.F. Müller & $n \cdot \mathbf{n}$ & $\mathrm{n} \cdot \mathrm{n}$ & 0.7 \\
\hline lahrata pisc. alpestros Küuster & 35 & 3745 & 149 \\
\hline Buhinia temaculata $\mathrm{L}$ & 0.6 & n.n & n. n \\
\hline Aithininia Opercula & 118 & n.n & n.n \\
\hline Radix auricularia L. & $n \cdot n$ & 105 & $\mathbf{n} \cdot \mathbf{n}$ \\
\hline Radix ovala Drap & 35 & 8.2 & 180 \\
\hline Planorbis carinatux O.F Müller & 10 & n.n & n.n \\
\hline Bathramphalus contorus L. & 0.3 & n.n & n. n \\
\hline (ivraulus acronicus Fer. & 30 & 212 & n.n \\
\hline Irmiger crista $\mathrm{L}$ & n.n & 52 & 3.9 \\
\hline llippeufis complanatus $\mathrm{L}$. & 5 & $n . n$ & n.n \\
\hline Prsidium sp. & n.n & 1312 & 259 \\
\hline Sphaerium carneum $\mathrm{L}$. & n.n & 0.3 & n.n \\
\hline Ib) Landmollusken : & n.n & $\mathrm{n} \cdot \mathrm{n}$ & n.n \\
\hline Carrchium munmmu O.F Müller & 60 & $n, n$ & n.n \\
\hline Cochlicopa luhrica OF Müller & n. n & n. n & 0.7 \\
\hline Iallonia pulchella O.F. Müller & 60 & n.n & n.n \\
\hline Ena ohscura O.F. Müller & 10 & n.n & n.n \\
\hline Zonitoides miridus O.F. Müller & 5 & $n, n$ & $\mathrm{n}, \mathrm{n}$ \\
\hline \multicolumn{4}{|l|}{11 Insekten reste [Reste/hg TS]: } \\
\hline Plateumaris stricea & 15 & $n, n$ & n.n \\
\hline Donacia (Puppenhüllen) & 100 & $n \cdot n$ & n.n \\
\hline llvdrophilidoe n.b. & 2 & n.n & $n, n$ \\
\hline Truchoptera (Gebäuse) & 10 & n.n & n.n \\
\hline
\end{tabular}

Tab. 4: Ergebnisse der Wirbellosenbestimmungen ausgewählter Proben. Untersucht wurde die Fraktion 0,63 bis 6,3 mm (Grobsand + Feinkies). (Datierungen vgl. Tab. 3). 
Das Artenspektrum der Mollusken besteht ausschließlich aus submers lebenden Formen; im Gegensatz zur vorherigen Probe fällt hier die hohe Individuendichte auf. Es dominieren mit der Wasserschnecke Valvata piscinalis alpestris und Muscheln der Gattung Pisidium Sippen des Bewegtwassers, während Formen, die in pflanzenreichen, stehenden oder langsam fließenden Gewässern leben, wie Gyraulus acronicus, Radix auricularia, $R$. ovata und Armiger crista deutlich schwächer vertreten sind.

Der insgesamt recht geringe Gesamtkarbonatanteil setzt sich überwiegend aus klastischem Karbonat zusammen, in dem der Mg-Molenbruch sehr hoch liegt (vgl. Ostendorp 1990:78). Der hohe Glühverlust erklärt sich entweder durch Einschwemmung von humosem Oberbodenmaterial oder durch die in situ-Primärproduktion höherer Wasserpflanzen.

Auch hier handelt es sich vermutlich um zunächst fluvial erodiertes Material, welches an dieser Stelle unter strömungsärmeren Bedingungen wieder abgesetzt worden ist. Zu denken wäre an Hochflut-Spülgut, das im Randbereich einer wassererfüllten vegetationsreichen Rinne liegengeblieben ist.

Das Material aus G3SW/A-2.1 dagegen deutet auf rein lakustrische Ablagerungsbedingungen in strömungsarmem Milieu hin. Die Hauptmenge des Siebrückstandes besteht aus Characeen-Krusten. Diese bilden zusammen mit den ebenfalls sehr reichlich vorhandenen Characeen-Oogonien die Reste der ehemals wahrscheinlich den gesamten Gewässergrund bedeckenden Armleuchteralgenrasen. Andere Wasserpflanzen-Samen sind nicht vertreten. Auf Stillwasserbedingungen weist der vergleichsweise hohe Anteil von Ostracoden-Schalen hin. Terrestrische Partikel, z.B. Grobdetritus oder Gesteinstrümmer treten demgegenüber stark zurück.

In der artenarmen Molluskenfauna dominieren Muscheln der Gattung Pisidium sowie die Wasserschnecken Radix ovata und Valvata piscinalis alpestris. Die genannten Arten leben ausschließlich submers und hier vornehmlich in bewegtem Wasser, während die weniger häufig vorhandene Wasserschnecke Valvata cristata bevorzugt in Pflanzenbeständen stehender Gewässer vorkommt. Die gefundenen Süßwasserschnecken aus dem $\mathrm{B} \emptyset$ lling und Aller $\varnothing \mathrm{d}$ sind typische Vertreter einer spätglazialen Molluskenfauna (JAECKEL 1956, KowALKowSKı \& BERGER 1972, LozeK 1965 und 1976; MARCUSSEN 1967, SPARKS 1962).

Der Gesamt-Karbonatgehalt ist mit $936 \mathrm{~g}^{*} \mathrm{~kg}^{-1} \mathrm{TS}$ sehr hoch. Der niedrige $\mathrm{Mg}-\mathrm{Molenb}$ bruch weist auf rein biogen gefällte Karbonate hin.

Bei dem Ablagerungsraum handelt es sich um eine flache, ausgedehnte Senke, die vermutlich geringfügig durchströmt war. In dieser Senke wuchsen dichte Characeen-Rasen, die durch biogene Kalzitfällung für die bis zu $70 \mathrm{~cm}$ mächtigen Seekreideablagerungen sorgten. 


\section{Diskussion}

\subsection{Datierung der Flußablenkung}

Im Einklang mit früheren Arbeiten zur regionalen Quartärgeologie (SCHREINER 1974 und 1989) sowie gestützt auf eigene geomorphologische Befunde (vgl. BLum 1993) muß davon ausgegangen werden, daß der Eisrandstausee, der sich vor dem zurückweichenden westlichen Rheingletscher im Bereich des Bohlinger- sowie des Zeller-See-Beckens gebildet hatte, zunächst über das Durchbruchstal bei Rielasingen-Arlen entwässerte. Dabei schnitt sich der Schmelzwasserstrom in die Schotterebene des Standes 7 ein und schuf sich auf diese Weise ein bis zu $300 \mathrm{~m}$ breites, heute noch morphologisch auszumachendes Glazialtal (vgl. Abb. 3: „SW-Tal“). Die Radolfzeller Aach, die zu jener Zeit bereits ein selbständiges Gerinne von ca. $18 \mathrm{~km}$ Länge darstellte, erreichte im Bereich des Ortskerns von Rielasingen den o.g. Schmelzwasserstrom und folgte dem gemeinsamen südwestlich gerichteten Abflußweg bis zur Mündung in die Hochrheinrinne, ca. $2 \mathrm{~km}$ westlich von Hemishofen.

Nachdem nun die Ostflanke des Höri-Hügellandes eisfrei geworden war und damit eine tiefergelegene Entwässerungsmöglichkeit über Stein am Rhein offenstand, muß der Schmelzwasserstrom im Bereich RielasingenArlen allmählich versiegt sein, so daß der Aach das inaktiv gewordene Glazialtal allein überlassen blieb (Abb. 9 a).

Die weitere Entwicklung des SW-Tales ist durch ein dreigliedriges Prozeßgeschehen gekennzeichnet:

1. Einschneidung der SW-Rinne in das nicht mehr schmelzwasserführende Glazialtal durch die Aach.

2. Abklingen der fluvialen Aktivität der Aach und damit verbunden, die allmähliche Verfüllung der SW-Rinne mit Feinsedimenten.

3. Ende des aktiven Fließgeschehens und anschließendes Torfwachstum im Grundwasserschwankungsbereich.

Der erste Prozeß, die Rinnen-Eintiefung, läßt sich zeitlich nicht genau bestimmen; er dürfte zu Beginn des Standes 8 des Untersee-Gletschers in Gang gekommen und spätestens zu Beginn der Feinmaterial-Ablagerung im Rinnenzentrum beendet gewesen sein. Da die Kurzkerne bestenfalls die obersten $70 \mathrm{~cm}$ des rinnenfüllenden Mineralsedimentes erfassen und zeitlich bereits in die Älteste Tundrenzeit zurückreichen (vgl. G3SW/B in Abb. $6 \mathrm{f}$ ), ist es denkbar, daß die tieferliegenden Schichten der Rinnenverfüllung noch ins pollenfreie Spätwürm datieren.

Maßgebend für die Datierung der Laufänderung aber ist der Wechsel von den am Top der SW-Rinne liegenden jüngsten minerogenen Rinnense- 
dimenten zu der aufliegenden Torfschicht. Damit kommen den Pollendatierungen aus den Gruben G2SW/A und G3SW/B sowie den Radiokarbonergebnissen aus G2SW/A-1.5 (basale Torfschicht im Bereich einer Rinnenflanke) und G3SW/B-1.3 ausschlaggebende Bedeutung zu.

In G3SW/B setzt die Torfbildung bereits im beginnenden Allerød (PZ II; ca. $11800 \mathrm{BP}$ ) ein. Die obersten lithologischen Einheiten des basal anschließenden minerogenen Sediments (LE 2.1.1 und 2.1.2) datieren noch in die Bøllingzeit/Ältere Tundrenzeit (PZ Ib/Ic; ca. $12500 \mathrm{BP})$. Bei G2SW/A findet der Schichtübergang im Bereich der PZ II/III (Allerød/ Jüngere Tundrenzeit, ca. 10800 Jahre BP) statt. Ob die letztgenannten Proben tatsächlich etwas jünger sind als im ersten Fall, oder ob vom Hangenden her nachträglich jüngere Pollen eingeschwemmt worden sind, kann zunächst rein pollenanalytisch nicht entschieden werden. Das vorliegende ${ }^{14} \mathrm{C}$-Datum aus derselben Grube (G2SW/A) (vgl. Tab. 2 und Abb. 6e) datiert eine Holzprobe, die etwas oberhalb der Torfbasis geborgen wurde, auf 10040 BP und stimmt daher gut mit der Annahme eines etwas geringeren Pollenalters überein. Da dieses Datum auch nicht mit den Pollendatierungen aus der Grube G3SW/B im Widerspruch steht - der in diesen Dimensionen eher geringe Zeitunterschied kann auch mit den nicht in beiden Fällen jeweils exakt rinnenzentral gelegenen Beprobungsstellen erklärt werden -, soll es zur zeitlichen Festlegung der Laufablenkung herangezogen werden.

Das ${ }^{14} \mathrm{C}$-Datum aus G3SW/B-1.3 von $1380-1223 \mathrm{BC}$ muß als nicht schichtdatierend verworfen werden, da es in klarem Widerspruch zu sämtlichen Pollenbefunden aus der SW-Rinne sowie zu dem o.a. ${ }^{14} \mathrm{C}$-Alter steht. Die Ursache dieser offensichtlichen Fehlbestimmung ließ sich leider nicht mehr rekonstruieren.

Da die Transekte T2 SW und T3 SW, abgesehen von der oben beschriebenen Hauptrinne, keine weiteren durchgehenden Sekundärrinnen und auch keinerlei Erosionsdiskordanzen aufgedeckt haben, dürfen weitere Verlagerungen der generellen Abflußrichtung, also ein mehrfacher Richtungswechsel der Unteren Radolfzeller Aach zwischen dem Untersee- und Hochrhein-Mündungsgebiet, wie er von SchlichtHerLe (1985:27) zur Diskussion gestellt wurde, mit hoher Wahrscheinlichkeit ausgeschlossen werden.

Zusammenfassend bleibt festzuhalten, daß der Laufrichtungswechsel der Radolfzeller A a ch eindeutig ins Spätglazial fällt. Er dürfte etwa im Übergang Allerød/Jüngere Dryas, also vor etwa 11000 Jahren stattgefunden haben. 
ERB's (1949) vorwiegend morphologisch gestützte Hypothese, die Ostablenkung der Aach datiere ins Atlantikum, oder sei jünger, muß also vor dem Hintergrund der vorliegenden Befunde revidiert werden. Als Hauptargument für seine jüngere Datierung der Aachablenkung führte er seinerzeit an, daß, ca. $1 \mathrm{~km} \mathrm{SW}$ der Ortschaft Moos, die hier nach Osten gegen den Mooswald hin ausbuchtende Flußniederung eine Erosionsleistung der Aach darstelle und somit als relativ jünger im Vergleich zum umgebenden Ausläufer des Schiener Berg-Schwemmfächers betrachtet werden müsse. Diesen aber spricht er, aufgrund eingeschalteter Tonlagen, als sublakustre Bildung an. Den Zeitpunkt des Trockenfallens des Schwemmfächers setzt er nun mit dem Alter von angeblich mesolithischen Silexgerätschaften, welche oberhalb der Flußniederung gefunden wurden, gleich. Deshalb also müsse die Aachablenkung frühestens zum Zeitpunkt der mesolithischen Besiedlung in diesem Gebiet oder später erfolgt sein. Obwohl die auch von uns überprüften morphologischen Befunde eindeutig sind, ist die chronologische Verknüpfung in sich nicht schlüssig. Denn aus der mesolithischen Kulturtätigkeit des Menschen an dieser Stelle kann nur gefolgert werden, daß der Schwemmfächer zu jenem Zeitpunkt bereits trockengelegen haben muß. Wie lange dies allerdings schon der Fall gewesen sein mag, geht aus dem Argument nicht hervor. Also kann die angeführte Erosionsspur der Aach auch beträchtlich älter sein als das angegebene mesolithische Maximalalter. Überdies sind nach heutigem Kenntnisstand wahrscheinlich sämtliche Silexfunde dem Neolithikum zuzuordnen (B. DieckManN, Landesdenkmalamt Hemmenhofen, mündl. Mitt.; vgl. auch DiecKmanN 1990:109).

\subsection{Spezielle Verlandungsbildungen im Randbereich des SW-Tals}

Etwa $1 \mathrm{~km}$ nördlich von Ramsen befindet sich eine ca. $1 \mathrm{~km}^{2}$ große, in die Schotterflur eingetiefte flache Depression, deren Südflanke in das Glazifluvialtal übergeht (Gewanne Ried, Chollbrunnen, Seewadel; vgl. Abb. 3). Die heutige Sohle liegt mit ca. $415 \mathrm{~m}$ NN rund $2 \mathrm{~m}$ unterhalb des umgebenden Terrassenniveaus. Im Zentrum der Hohlform liegen noch flach inselartig aufragende Reste (Gewann „Hinterbol“), die sich aufgrund ihrer Höhenlage und lithologischen Zusammensetzung mit den randlichen Terrassenschottern verknüpfen lassen. Im Zuge der geomorphologischen Geländeaufnahme traten in Bereichen frisch gepflügter Äcker stellenweise karbonatreiche Feinsedimente zu tage, die von ihrer faziellen Ausprägung her Ähnlichkeit mit der in T2 SW angetroffenen Seekreide aufwiesen. Stratigraphisch sind sie in das Liegende der anmoorigen Deckschichten 
einzuordnen. In den Tieflagen der Mulde stehen oberflächlich zumeist Niedermoortorfe an. Diese wurden bis ins 20 . Jahrhundert abgebaut und wirtschaftlich genutzt. (Das Gebiet war bis ins 18. Jh. Allmende der Gemeinde Rielasingen. Von 1837 bis 1919 ist gewerbliche Torfstecherei in größerem Umfang belegt; bis 1948 fand noch vereinzelt privat betriebener Torfabbau in kleinerem Rahmen statt (STREIT 1993:138).

Die Genese der Depression ist ungeklärt; möglicherweise handelt es sich um ein Toteisfeld, das beim Rückzug des Gletschers vom Stand 6 zum Stand 7 liegen blieb. Im Zusammenhang mit der im SW-Tal anstehenden Seekreide mit einer Schichtoberkante von etwa 413,5 m NN (vgl. Abschn. 3.4 sowie Abb. 5 c und 6 f) könnte gefolgert werden, daß die Hohlform zumindest im Spätglazial wassererfüllt war. Das heißt, daß an dieser Stelle möglicherweise ein schwach durchströmtes oder stehendes Gewässer mit einer ungefähren Spiegellage von $414 \mathrm{~m} \mathrm{NN}$ existiert hat. Ob und wie lange es nach dem Laufrichtungswechsel der Aach fortbestanden hat, müßte durch neu angesetzte und weitergehende Untersuchungen geklärt werden.

Schwierig gestaltet sich auch die Deutung des Steinpflasters (G3SW/ A-3.1), das im Liegenden der Seekreide aufgeschlossen wurde (vgl. Abschn. 3.2). Es wird angenommen, daß die Entstehungzeit des Steinpflasters ebenso wie die des unterliegenden Silikatsandes in das pollenfreie Spätwürm fältt, da beide Schichten nicht pollenführend waren (vgl. Abb. $6 \mathrm{f}$ ). Die Steine entstammen Moränen- bzw. Schotterablagerungen und wurden erst sekundär in diese Position gebracht. Als Transportmedium kommen Eisschollen in Frage, in die die Steine eingefroren waren. Ein fluvialer Transport erscheint aufgrund der beträchtlichen Größe der Steine unwahrscheinlich. Die scharfen Bruchkanten entstanden vermutlich in situ, z.B. durch Frostsprengung.

\subsection{Die Entwicklung des Ostabflusses}

Die Interpretation der hydrographischen Entwicklung des Ostabflusses nach der Aach-Ablenkung um $11000 \mathrm{BP}$ stützt sich vornehmlich auf die granulometrischen Befunde. Die im Bereich der Transekte T1 SW, T1 E und $\mathrm{T} 2 \mathrm{E}$ bereits morphologisch erfaßten Rinnenabschnitte ließen sich stratigraphisch bestätigen (vgl. Abschn. 3.2 sowie Abb. 6 a - d). Die glazifluvialen Kiese, die auch hier die Sohlbasen bilden, lassen sich zwanglos zwischen den Randgruben verbinden, so daß die Rinnen als spätere Bildungen angesprochen werden müssen. Die an organischen Resten reichen Feinsedimentlagen der zentralen Rinnenbereiche zeugen von der nachlassenden Transportkraft des strömenden Wassers und werden deshalb als Verlandungssedimente interpretiert. 
Ein Teil dieser Rinnenelemente läßt sich über Parallelisierung von lithologisch ähnlichen Einzelproben entlang der Gefällelinie von G1SW bis G2E verknüpfen. Demzufolge ziehen insgesamt drei zusammenhängende „Sedimentspuren“ ausgehend von G1SW am Aachknie über G1E und G2E weiter ostwärts. Zwei dieser Spuren sind charakterisiert durch hohe granulometrische Ähnlichkeit der zugehörigen Sedimentproben. Die dritte zeichnet sich durch das Vorkommen onkolithischer Krümelkalke aus.

Spur 1: Der in G1SW/B-2.1 angetroffene humose Lehm (feinsandiger Grobschluff) läßt sich in jeweils rinnenbasisnaher Lage über G1SW/E-3 und G2E/A-4 unter ostwärts gerichtetem Gefälle verfolgen. Sowohl die Korngrößenverteilungen (Modalwerte: $-1,63$ bis $-1,65 \zeta$ ) als auch die $\mathrm{Ge}-$ halte an organischem Material (18,5-22,9\% der Trockensubstanz) liegen sehr eng zusammen (vgl. Abschn. 3.4).

Spur 2: Eine zweite Spur verläuft von G1SW/B-2.2 über G1SW/E-1.2 nach G1E/B-3. Auch hier stimmt die Korngrößenverteilung (Modalwerte $-1,63$ bis $-1,94 \zeta$; Grobschluff mit starkem Mittelschluffanteil) gut überein. Die Gehalte an organischer Substanz schwanken nur wenig und liegen im Mittel bei $9,2 \%$.

Spur 3: Ein Band aus onkolithischen Krümelkalken zieht, ebenfalls beginnend in G1SW, durch beide östlichen Grubenkomplexe. In G1SW/E-2 tritt es als schlecht sortiertes, heterogenes Gemenge zwischen den im Feinkies-Korngrößenbereich liegenden Kalkkonkretionen und dem eher schluffigen Abrieb in Erscheinung. In G1E/C-3 liegt das Kalksediment als sehr gut sortierter Sand, ebenfalls in Sohlnähe des Rinnenzentrums vor. Es ist also davon auszugehen, daß eine Transportsortierung stattgefunden hat. Die Hauptursache für die abnehmende Korngröße dürfte im Abrieb der mechanisch wenig widerstandsfähigen Grobpartikel liegen. In G2E/A-5 nehmen allerdings die kiesigen Anteile wiederum deutlich zu.

Diese 3 Spuren verlaufen nicht unabhängig voneinander, sondern zeichnen unter ostwärtigem Basisgefälle denselben Weg nach. Sie werden daher unter dem Begriff „Ostrinne I" zusammengefaßt und als älterer Aachlauf gedeutet (Abb. 9 b). Sechs ${ }^{14} \mathrm{C}$-Daten stehen zur Verfügung, um den Zeitpunkt des Inaktivwerdens der Ostrinne I zu diskutieren: Ein Datum gehört zu G1E/B-3; es repräsentiert einen rinnenbasisnah gelegenen Schluff und datiert auf 1380-1228 BC. Die beiden Proben aus G2E/B-2 liegen zwischen 821 und $542 \mathrm{BC}$. Allerdings stammen sie aus der überlagernden Torfschicht und markieren daher eine Situation, die bereits in die Zeit nach der Rinnenverlandung fällt. Die wegen der schlechten Pollenerhaltung nur unscharf auflösenden Pollenprofile aus der Grube G2E/A decken sich mit 
den letztgenannten Radiokarbondatierungen; dem Schichtwechsel Rinnensediment/Torf entsprechen die Pollenzonen VIII/IX (Subboreal/Älteres Subatlantikum, ca. 3500 bis 2500 Jahre vor heute). Die restlichen drei ${ }^{14} \mathrm{C}$-Proben aus den Gruben G1E/C und G1E/D haben sich sämtlich als neuzeitlich erwiesen (17. Jh. AD oder jünger). Da sie also wesentlich jünger sind als der sicher verlandungsindizierende Torf aus G2E/A und zugleich rinnenperipher liegen, werden sie nicht mit dem Inaktivwerden der Ostrinne I in Zusammenhang gebracht. Damit ergibt sich für die Interpretation des von T1 E gequerten Rinnenabschnittes ein differenziertes Gesamtbild: Die stratigraphisch am tiefsten gelegenen, bronze- bis eisenzeitlich datierten Sedimente, sind der Ostrinne I zuzuordnen. Die neuzeitlich datierenden Proben könnten anthropogenen Ursprungs sein; d.h. im Zuge der Industrieansiedlung im 19. Jh. wurde der Aachlauf künstlich nordwärts verlegt, wobei die alte Rinne verfüllt wurde. Ob aber das alte Gerinnebett nach dem Inaktivwerden der Ostrinne I wenigstens in dem von T1 E erfaßten Teilabschnitt kontinuierlich bis in die Neuzeit wasserführend war (für den Abschnitt innerhalb T2 E kann dies ausgeschlossen werden) oder zu einem späteren Zeitpunkt an dieser Stelle reaktiviert wurde, kann nicht entschieden werden.

Abschließend bleibt also festzuhalten, daß aufgrund der o.g. ${ }^{14} \mathrm{C}$-Daten (vgl. auch Abb. $6 \mathrm{c}$ und d sowie Tab. 2) die Zeitspanne, in der die Inaktivierung der Ostrinne I erfolgt sein muß, auf max. $1380 \mathrm{BC}$ bis $542 \mathrm{BC}$ eingegrenzt werden kann. Damit fällt dieses Ereignis in die Spätbronzezeit oder in die frühe Eisenzeit und somit in eine Phase, in der das gesamte Aachgebiet bereits intensiv von ackerbäuerlichen Kulturen besiedelt war.

Eine weitere, sich morphologisch abzeichnende Rinne, konnte über die Gruben G2E/E und G2E/F im Flankenbereich stratigraphisch erfaßt werden. Hier war es jedoch nicht, wie im Falle der Ostrinne I, möglich, Sedimentspuren, die von G1SW über G1E bis hin zu G2E durchziehen, zu identifizieren. Möglicherweise verlief diese Rinne in den Bereichen von G1SW und G1E anders, oder die ursprünglich vorhandenen Sedimentspuren wurden durch späteren Abtrag ausgelöscht. Da aber aufgrund der Schichtverläufe in G2E/E die Rinnennatur als gesichert gilt, und da infolge der im Vergleich zum Aachknie deutlich tieferen Lage der Rinnensedimente ebenfalls ein ostwärts gerichtetes Gefälle bestanden haben muß, wird auch diese Rinne als ehemaliger Aachlauf gedeutet und als „Ostrinne II" bezeichnet (Abb. 9 b). Ob sie allerdings älter ist als Ostrinne I und so möglicherweise den primären Abflußweg nach der Ablenkung repräsentiert, muß vorläufig offen bleiben. Es liegt zwar ein ${ }^{14} \mathrm{C}$-Datum aus G2E/ E-2.4 mit 2452-2202 BC vor; es ist aber zweifelhaft, ob dieser Wert zur 
Schichtdatierung herangezogen werden kann, da es sich bei der sehr schlecht erhaltenen Probe möglicherweise um (nicht schichtdatierendes) Wurzelholz gehandelt hat.

\subsection{Mögliche Ursachen des Laufrichtungswechsels}

Bei der Diskussion der Ursachen des Laufrichtungswechsels müssen zwei Faktorenkomplexe unterschieden werden: (1) die flußmorphologischen und hydrogeologischen Gegebenheiten im Bereich des Aachknies nach Rückzug des Zeller See-Lobus des westlichen Rheinvorlandgletschers, und (2) das hydrologische Regime und damit verknüpft, die Dynamik des Sedimenttransportes vor dem Hintergrund spätglazialer Klimaschwankungen.

1. Nach Rückzug des Zeller See-Lobus bestand für die Aach bei Rielasingen ein Gefälle-Vorteil in Richtung auf das glazial ausgetiefte, östlich gelegene Zungenbecken. Abb. 8 a zeigt den Sohlverlauf der heutigen Aach im Längsschnitt und im Vergleich dazu die Tallinie des oberhalb des Aachknies ansetzenden SW-Trockentales. Bei der heutigen Aachrinne ist eine deutliche Gefällsversteilung im Bereich zwischen Rielasingen und Worblingen auszumachen. Ab Worblingen nimmt das Gefälle wieder ab und liegt in der Größenordnung wie oberhalb der Versteilung. Das Gefälle des SW-Tales ist deutlich geringer als das des heutigen Ostverlaufs und entspricht demjenigen der Aach oberhalb Rielasingen.

In der Abb. 8 b und $c$ werden die Sohlniveaus der einzelnen Transekte miteinander verbunden und verglichen. Dabei wurden als Kriterium die Top-Niveaux der Rinnenfüllungen herangezogen, da sie das Ende des fluvialen Geschehens indizieren. Bei $\mathrm{T} 1 \mathrm{~N}$ hingegen mußte ersatzweise die heutige Sohlhöhe verwendet werden. Das Gefälle der gegenwärtigen Aachrinne schwankt zwischen 4,53 und 3,00\% (Mittelwert 3,81\%o); die Gefällsdaten der SW-Rinne liegen mit 1,65 bzw. 1,14\% (Mittelwert $1,46 \%$ ) wesentlich tiefer. Das geringste Sohlgefälle herrscht dabei zwischen T2 SW und T3 SW.

Im Untergrund der Umgebung des Aachknies stehen weiträumig hydraulisch hochdurchlässige Grobkiese mit geringem Feinsedimentanteil an. Daher kann angenommen werden, daß der eigentlichen Laufablenkung über einen längeren Zeitraum ein über Uferexfiltration aus der Aach gespeister Grundwasserstrom in Richtung Osten vorausging. Ob allerdings, wie ERB (1949) in seiner Arbeit anführt, durch das weiter östlich zwangsläufig wieder zutage tretende Grundwasser eine nennenswerte rückschreitende Erosion geleistet werden konnte und damit die Aachablenkung durch „Flußanzapfung“ erklärt werden kann, ist sehr zweifelhaft. Denn es ist schwer vorstellbar, daß Quellen im quasiebenen Gelände - und solche 
Verhältnisse müssen ja aufgrund der früheren glazifluvialen Überformung im Durchbruchstal von Worblingen angenommen werden - ihre Lage erosiv zurückverlegen.

Vielmehr sollte den rinnenstratigraphischen Verhältnissen innerhalb der SW-Rinne Beachtung geschenkt werden: Die mächtige Feinsedimentverfüllung beweist, daß die Aachablenkung eine längere Vorgeschichte gehabt haben muß. Das heißt, daß die aufgrund des geringen Gefälles und der relativ großen Sohlbreite bereits erwähnten abflußträgen Bedingungen im alten SW-Tal schließlich dazu geführt haben, daß der Fluß einen Teil seiner Sedimentfracht absetzen mußte und daher sein eigenes Sohlniveau höhergelegt hat. Diese Vorstellung kann auch zwanglos mit der These der nachlassenden Wasserführung des Flusses infolge des Grundwasser-Abstromes im Bereich der späteren Ablenkungsstelle verknüpft werden; denn mit dem Wasserverlust ist eine weitere Minderung der Transportkraft und damit eine zusätzliche Erhöhung der Sedimentationsrate im alten Flußbett verbunden. Schließlich war eine kritische Situation erreicht, bei der, vermutlich durch Hochwasserereignisse, die damals ohnehin flache Wasserscheide zum Bohlinger Becken überschritten wurde und durch nachfolgende erosive Eintiefung der östliche Abflußweg endgültig freigelegt werden konnte. Somit war die Aachablenkung vermutlich nicht allein das Ergebnis eines singulären Extremereignisses, sondern letztlich das Resultat einer längerwirkenden, vorwiegend gerinnehydraulisch und hydrogeologisch bestimmten Prozeßkette.

Auf der Grundlage der diskutierten rinnenmorphologischen und rinnenstratigraphischen Verhältnisse muß auch die Ansicht ScHMIDLE's (1914:70), die Aach würde von einer WNW-ESE streichenden Verwerfung abgelenkt, als unwahrscheinlich angesehen werden. Darüberhinaus wäre die

Abb. 8: Gefällsverhältnisse der heutigen Aachrinne im Vergleich zur spätglazialen SW-Rinne.

(a) Längsschnitt des Gefälleverlaufes im Bereich des Aachknies (Abszissennullpunkt entspricht der Position des unweit der Aach gelegenen Singener Bahnhofes. Der Endpunkt der SW-Linie markiert das Zusammentreffen des SW-Trockentales mit der Biber, westlich von Ramsen. Bei der heutigen Aach-Rinne liegt er ca. $1 \mathrm{~km}$ westlich der Ortsmitte von Bohlingen).

(b) Gefällewerte $\left(\mathrm{m}_{\mathrm{i}}\right)$ der heutigen Aachrinne zwischen den Transekten T1 N, T1 SW und T2 E (bei T1 SW wurde ersatzweise das einnivellierte Sohlniveau der unweit des Transektes verlaufenden Flußrinne verwendet).

(c) Gefällewerte $\left(m_{i}\right)$ der SW-Rinne zwischen den Transekten T1 N, T2 SW und $\mathrm{T} 3 \mathrm{SW}$. 


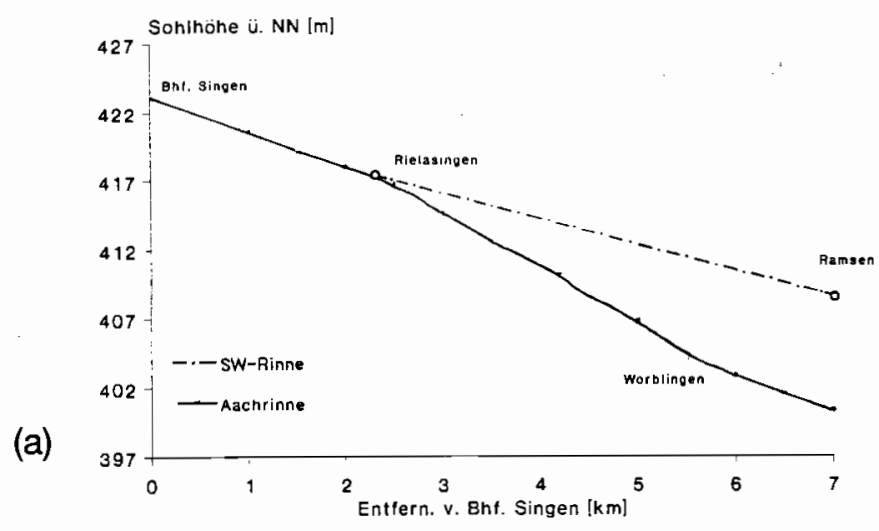

(b)
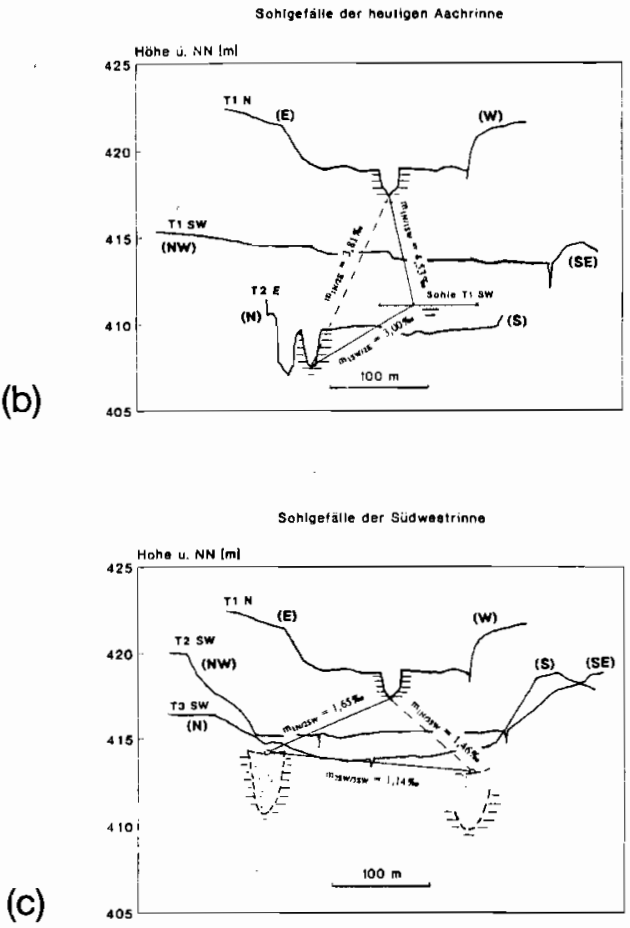
flußmorphologische Wirksamkeit dieser Störung, deren Verlauf im Bereich der Ablenkungsstelle nach wie vor nicht genau bekannt ist (vgl. SCHREINER 1989: 66f.), durch die mehrere Dekameter betragende glazialsedimentäre Überdeckung längst aufgehoben.

2. Die Frage nach den paläoklimatischen Faktoren, die für den Laufrichtungswechsel von ursächlicher Bedeutung gewesen sein könnten, impliziert die Frage nach der Variabilität des Abflusses und des Sedimentdargebotes im Zusammenhang mit der letztlich auch vom Klima abhängigen Dichte der Vegetationsbedeckung.

Für die Älteste Tundrenzeit kann von einer kalt-trockenen Klimaphase und einer dementsprechend offenen Pioniervegetation aus niederwüchsigen Kräutern, später auch mit Zwerggehölzen, ausgegangen werden (Frenzel 1983b, Welten 1982, Zoller 1987). Die schüttere Vegetationsdecke dürfte den Niederschlag nicht nennenswert zurückgehalten und somit den Oberflächenabtrag stark begünstigt haben. Der Übergang zum Bølling um ca. $12600 \mathrm{BP}$ ist durch eine rasche Erwärmung und Vegetationsverdichtung gekennzeichnet, wobei vermutlich auch die Niederschlagsmenge deutlich anstieg (LOTTER \& Z BINDEN 1989, VAN GeEl \& KolSTRUP 1978, KAISER 1993). Das klimatische Optimum fiel gemäß der Interpretation von $\delta{ }^{18} \mathrm{O}$-Messungen in das Bølling, während es im Allerød bereits feuchter und kälter wurde. Die nun aufkommenden Birken- und Kiefernwälder dürften die Landoberfläche wesentlich besser vor erosivem Angriff geschützt haben. In dieses erste späteiszeitliche Klimaoptimum fallen auch die Seekreideablagerungen im T3 SW, die bereits ein Nachlassen der fluvialen Aktivität in diesem Gebiet indizieren.

Der Klimarückschlag der Jüngeren Tundrenzeit (ca. 10 700-10 300 BP), der sich bereits in der letzen Hälfte des Allerøds ankündigte, war gekennzeichnet durch einen erheblichen Rückgang der Jahresmitteltemperaturen und einer weiteren Zunahme der Niederschlagsmengen, gefolgt von einer starken Auflockerung der Vegetationsdecke (Rösch 1990, VAN GEeL \& KolSTRUP 1978, ZolLER 1987). Sowohl der Oberflächenabfluß als auch das Sedimentdargebot dürften sich folglich gegenüber dem vorangegangenen Bølling/Allerød-Interstadial generell erhöht haben.

Für die Radolfzeller Aach bedeutet dies, daß sie, abgesehen von den ohnehin gefälleschwachen Bedingungen im SW-Tal, zusätzlich mit einer hohen Sedimentfracht belastet war, was die endgültige Verlandung zu Beginn der Jüngeren Tundrenzeit zusätzlich gefördert haben dürfte.

Damit zeigt die Radolfzeller Aach eine flußmorphologische Entwicklung, die von der zahlreicher anderer Flüsse abweicht (vgl. Becker 1982, SCHELlmann 1993). So fehlt eine späthochglaziale Sedimentakkumulation 

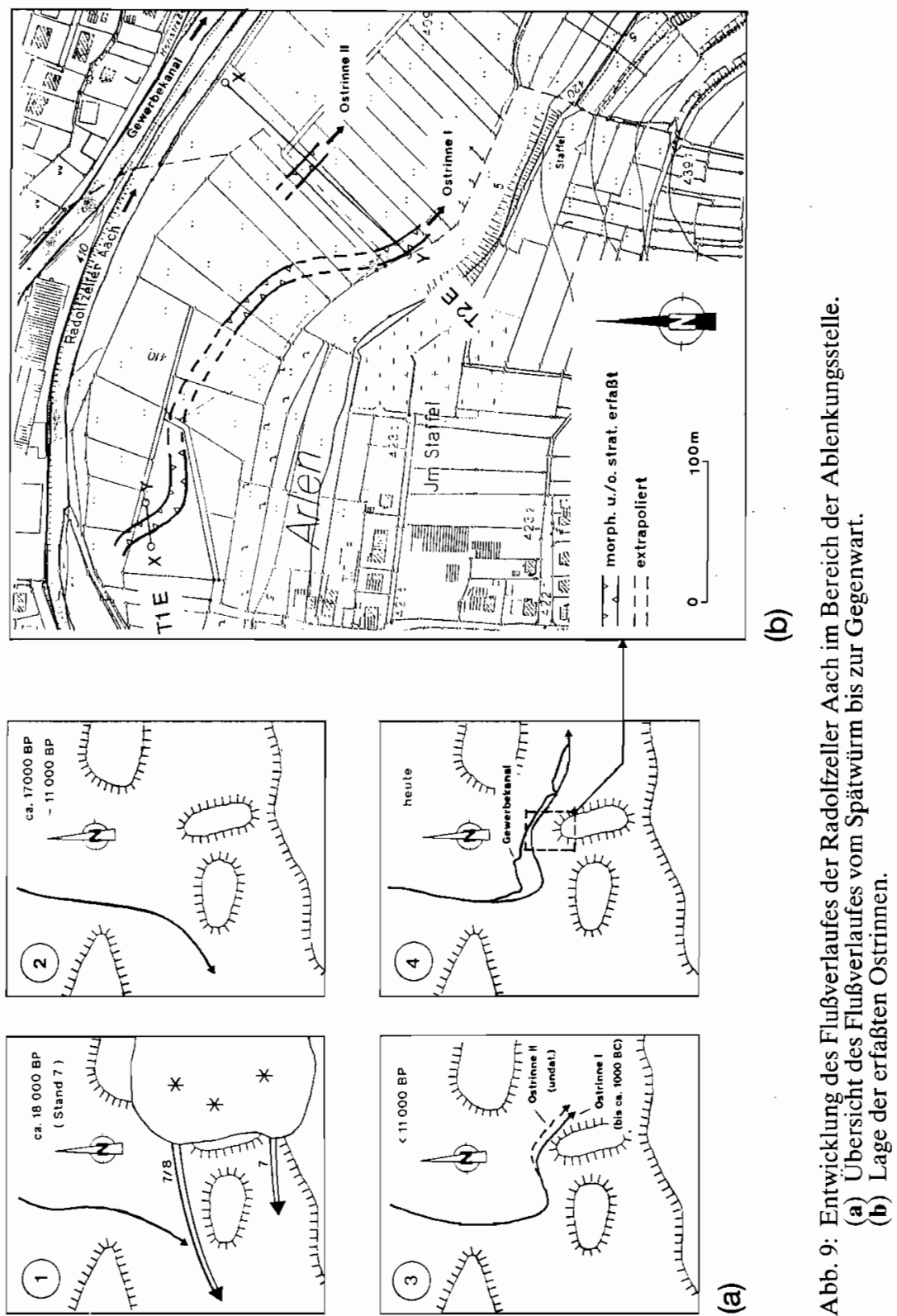
und eine bølling-allerødzeitliche Tiefenerosion, die der Niederterrasse $2 \mathrm{n}$. SchellmanN (1993) parallelisierbar wäre. Hier kommt es vielmehr zu einer Solerhöhung durch Stillwasserkalke. Der vermehrte Sedimenteintrag der Jüngeren Tundrenzeit, der an Donau, Isar, Weser, Main und Niederrhein zur Akkumulation der Niederterrasse 3 geführt hat (SCHELlmanN), ließ die SW-Rinne der Radolfzeller Aach endgültig verlanden.

Für die Zeit des älteren und mittleren Holozäns kann bezüglich der Konstanz des Gewässernetzes eine hydrologisch eher ruhige Phase angenommen werden, da für diesen Zeitabschnitt keine nennenswerten Lagevariationen der östlich ziehenden Rinnen festgestellt werden konnten. Günstigere Bedingungen in Bezug auf eventuelle Laufschwankungen waren erst wieder mit dem „Kälterückschlag“ im Subboreal gegeben und ganz besonders, nachdem der Mensch durch großflächige Rodungen hydrologisch verändernd in das Einzugsgebiet der Aach eingegriffen hatte.

Dank: Die Arbeit entstand innerhalb des DFG-Schwerpunktprogrammes „Siedlungsarchäologische Untersuchungen im Alpenvorland“. Der DFG und dem Landesdenkmalamt Baden-Württemberg, Abt. Bodendenkmalpflege sei für die finanzielle und logistische Unterstützung des Projektes gedankt. Unser Dank gilt auch den Grundstückseigentümern, die unsere „tiefgreifenden“ geologischen Aktivitäten auf ihrem Gelände duldeten. Herrn Dr. A. Billamboz danken wir für die Bestimmung der Holzproben. Nicht zuletzt gilt unser Dank auch Herrn Dr. Gerd SCH röDER (Institut für Seenforschung, Langenargen), der uns die Durchführung der laseroptischen Korngrößenanalysen ermöglicht hat.

\section{Schriften}

Becker, B.: Dendrochronologie und Paläoökologie subfossiler Baumstämme aus Flußablagerungen - Ein Beitrag zur nacheiszeitlichen Auenentwicklung im südlichen Mitteleuropa. - Mitt. Komm. Quartärforsch. Österr. Akad. Wiss., 5, 120 S., 34 Abb., 9 Tab., Wien 1982.

- An 11000 Year German Oak and Pine Dendrochronology for Radiocarbon Calibration. - Radiocarbon, Vol. 35 (1), 201-213, 9 Abb., New Haven (USA) 1993.

Bludau, W.: Zur Paläoökologie des Ammergebirges im Spät- und Postglazial. Diss., Univ. Hohenheim, 364 S., 61 Abb., 8 Tab., 24 Taf. i. A., Rheinfelden 1985.

- Ein Vergleich der aktuellen Vegetation mit dem Pollengehalt von Moosproben in der Birkenwaldstufe und der Tundra in Schwedisch-Lappland. Ber. Reinhold Tüxen-Ges., 6, [im Druck], Hannover 1994. 
Buum, H.: Flußtalgeschichte der Unteren Radolfzeller Aach. - Unveröff. Dipl.Arbeit, Univ. Freiburg i. Br., 110 S., 41 Abb., 9 Tab., 1 Beil., Freiburg 1993.

Erb, L.: Die Flußgeschichte der Radolfzeller Aach. - Mitt. Bad. Geol. Landesanst., 1949, 56-59, Freiburg i. Br. 1950.

Erdtman, G., Berglund, B. \& Praglowsk1, J: An introduction to Skandinavian pollen flora. - Grana Palynol. 2/3, 1-92, Stockholm 1961.

Firbas, F.: Spät- und nacheiszeitliche Waldgeschichte Mitteleuropas nördlich der Alpen. Bd. I: Allgemeine Waldgeschichte. - 480 S., 163 Abb., 28 Tab., (Fischer) Jena 1949.

- Spät- und nacheiszeitliche Waldgeschichte Mitteleuropas nördlich der Alpen. Bd. II: Waldgeschichte der einzelnen Landschaften. -256 S., 18 Abb., 39 Tab., (Fischer) Jena 1952.

Frenzel, B.: Zur Pollenanalyse von Lössen. - Eisz. u. Geg., 15, 5-39, Öhringen 1964.

- Die Vegetationsgeschichte Süddeutschlands im Eiszeitalter. - In: MüLlerBECK, H. [Hrsg.]: Urgeschichte in Baden-Württemberg, 91-166, 72 Abb., 6 Tab., (Theiss) Stuttgart 1983a.

- On the Central-European water budget during the last 15000 years. - Quarternary Studies of Poland 4, 45-59, 1 Abb., Warschau 1983b.

Frenzel, B. [Hrsg.]: Klimageschichtliche Probleme der letzten 130000 Jahre. 432 S., (Fischer) Stuttgart 1991.

JAECKEL, S.G.A.: Süßwassermollusken aus spät- und postglazialen Ablagerungen in Schleswig-Holstein. - Schr. Naturw. Ver. Schl.-Holst., 28 (1), 76-90, 1 Tab., Kiel 1956.

KaISER, F.: Ein späteiszeitlicher Wald im Dättnau bei Winterthur (Schweiz). - Diss. Univ. Zürich, 90 S., Zürich 1979.

- Beiträge zur Klimageschichte vom späten Hochglazial bis ins frühe Holozän, rekonstruiert mit Jahrringen und Molluskenschalen aus verschiedenen Vereisungsgebieten. - Eidgenöss. Forschungsanst. f. Wald, Schnee u. Landsch., 203 S., 97 Abb., 2 Tab., Birmensdorf (Schweiz) 1993.

KorTfunke, C.: Über die spät- und postglaziale Vegetationsgeschichte des Donaumooses und seiner Umgebung. - Diss. Bot., 184, 177 S., 30 Abb., 4 Tab., 11 Taf. i. A., Berlin/Stuttgart 1992.

Kowalkowskı, A. \& Berger, L.: Die Bedeutung der Conchylienfaunen für die Spätpleistozäne und Holozäne Sediment- und Bodenstratigraphie. - Bull. Soc. Amis Sci. Lett. Poz./D, 12/13, 215-224, 2 Abb., 2 Tab., 1 Taf., Poznan 1972.

LANG, G.: Zur späteiszeitlichen Vegetations- und Florengeschichte Südwestdeutschlands. - Flora, 139, 234-294, 11 Abb., 4 Tab., Jena 1952.

- Die Vegetation des Mindelsees und ihre Geschichte. - N.N.: Der Mindelsee bei Radolfzell. Monographie eines Naturschutzgebietes auf dem Bodanrück. - Natur- u. Landschaftsschutzgeb. Bad.-Württ., 11, 271-302, 18 Abb., Karlsruhe 1983.

- Die Vegetation des westlichen Bodenseegebietes. - 462 S., 41 Abb., 119 Tab., 16 Taf., (Fischer) Stuttgart/New York 1990. 
LOTTER, A. \& ZBInden, H.: Late-Glazial pollen analysis, oxygen-isotope record and radiocarbon stratigraphy from Rotsee (Lucerne), Central Swiss Plateau. Eclogae geol. Helv., 82, 191-202, 4 Abb., Basel 1989.

LozeK, V: Problems of Analysis of the Quaternary Nonmarine Molluscan Fauna in Europe. - Geol. Soc. Am., Special Paper, 84, 201-218, 3 Abb., 2 Tab., Prag 1965.

- Klimaabhängige Zyklen der Sedimentation und Bodenbildung während des Quartärs im Lichte malakozoologischer Untersuchungen. - Rozpr. CAVU, Rada, Matem. A Prir. Ved, Rocnik, 86, 3-108, 20 Abb., 9 Tab., Prag 1976.

Marcussen, I.: The Freshwater Molluscs in the Late-Glacial Deposits in the Bog of Barmosen, Southern Sjaelland, Denmark. - Medd. Danks Geol. Forend., 17 (2), 265-283, 4 Abb., 1 Tab., 1 Taf., Kopenhagen 1967.

OstendoRP, W.: Zur Stratigraphie und Sedimentpetrographie der Station Allensbach-Strandbad: Profilsäule E6. - Siedlungsarchäologie im Alpenvorland II. - Forsch. u. Ber. z. Vor- u. Frühgesch. Bad.-Württ., 37, 75-89, 2 Abb., 3 Tab., (Theiss) Stuttgart 1990.

- Hemmenhofener Methoden - Sedimentologische und paläolimnologische Methoden in der Siedlungsarchäologie. - Siedlungsarchäologie im Alpenvorland V, (Theiss) [im Druck], Stuttgart 1995.

Reimer, P. \& Stuiver, M.: Extended ${ }^{14}$ CData Base and Revised CALIB 3.0 ${ }^{14} \mathrm{C}$-Calibration Program. Radiocarbon, 35, (1), 215-230, New Haven (USA) 1993.

Rösch, M.: Ein Pollenprofil aus dem Feuenried bei Überlingen am Ried. Stratigraphische und landschaftsgeschichtliche Bedeutung für das Holozän im Bodenseegebiet. - Materialh. Vor- u. Frühgesch. Bad.-Württ. 7 - Ber. Ufer- u. Moorsiedl. SW-Deutschl., 2, 43-79, 7 Abb., 11 Tab., 2 Beil., (Theiss) Stuttgart 1985.

- Zwei Moore am westlichen Bodenseegebiet als Zeugen prähistorischer Landschaftsveränderungen. - Telma, 16, 83-111, 9 Abb., 3 Tab., Hannover 1986.

- Der Mensch als landschaftsprägender Faktor des westlichen Bodenseegebietes seit dem späten Atlantikum. - Eisz. u. Gegenw., 37, 19-29, 4 Abb., 1 Tab., Hannover 1987.

- Vegetationsgeschichtliche Untersuchungen im Durchenbergried. - Siedlungsarchäologie im Alpenvorland II. Forschungen u. Ber. zur Vor- u. Frühgeschichte in Bad.-Württ., 37, 9-64, 10 Abb., 3 Tab., (Theiss) Stuttgart 1990a.

- Veränderungen von Wirtschaft und Umwelt während Neolithikum und Bronzezeit am Bodensee. - Röm. germ. Komm., 71, 161-186, 20 Abb., 1 Tab., Mainz 1990b.

Schellmann, G.: La Structure Géomorphologique et Géologique des Fonds des Vallées dans les Domains subalpin et hercynien d'Allemagne. Revue Géographique de l'Est, 4, 235-259, 8 Abb., 1993. 
SchlichtHeRLe, H.: Prähistorische Ufersiedlungen am Bodensee - Eine Einführung in naturräuml. Gegebenheiten und archäolog. Quellen. - Materialh. Vor- u. Frühgesch. in Bad.-Württ. 7 - Ber. Ufer- u. Moorsiedl. SW-Deutschl., 2, 9-42, 15 Abb., (Theiss) Stuttgart 1985.

Schreiner, A.: Erl. geol. Kte. LK Konstanz (Bad.-Württ.) mit Umgebung 1: 50000. 2. berichtigte Aufl., 286 S., 28 Abb., 12 Tab., 11 Tafeln, 5 Beil., Freiburg i. Brsg. 1974.

- Erl. geol. Kte. Bad.-Württ., 1: 25000 Blatt 8219 Singen (Htwl.). 2. Aufl. mit Nachträgen. 139 S., 11 Abb., 10 Tab., 4 Tafeln, 8 Beil., Freiburg i. Brsg. 1989.

Sparks, B.W.: Post-Glacial Mollusca from Hawes Water Lancashire, Illustrating some Difficulties of Interpretation. - J. Conchology, 25 (2), 78-82, 3 Abb., 7 Taf., London 1962.

Strahm, C.: Die Vorraussetzungen für eine Chronologie der Feuchtbodensiedlungen in Südwestdeutschland. - Math. Vor- u. Frühgesch. Bad.-Württ. 7 - Ber. Ufer- u. Moorsiedl. SW-Deutschl., 2, 248-252, (Theiss) Stuttgart 1985.

VAN GeEl, B. \& Kolstrup, E.: Tentative explanation of the late glacial and early holocene climatic changes in north-western Europe. - Geol. Mijnb., 57 (1), 87-89, 1 Abb., Amsterdam 1978.

WeLten, M.: Stand der palynologischen Quartärforschung am schweizerischen Nordalpenrand. - Geogr. Helv., 2, 75-83, 3 Abb., 1982.

Zoller, H.: Zur Geschichte der Vegetation im Spätglazial und Holozän der Schweiz. - Mitt. Naturf. Ges. Luzern, 29, 123-149, 13 Abb., 1 Tab., Luzern 1987. 